\title{
MOD-DISCRETE EXPANSIONS
}

\author{
A. D. BARBOUR, E. KOWALSKI, AND A. NIKEGHBALI
}

\begin{abstract}
In this paper, we consider approximating expansions for the distribution of integer valued random variables, in circumstances in which convergence in law cannot be expected. The setting is one in which the simplest approximation to the $n$ 'th random variable $X_{n}$ is by a particular member $R_{n}$ of a given family of distributions, whose variance increases with $n$. The basic assumption is that the ratio of the characteristic function of $X_{n}$ and that of $R_{n}$ converges to a limit in a prescribed fashion. Our results cover a number of classical examples in probability theory, combinatorics and number theory.
\end{abstract}

\section{INTRODUCTION}

The topic of this paper is the explicit approximation, in various metrics, of random variables which, in terms of characteristic functions, behave like a sum

$$
X_{n}=Z_{n}+Y_{n}
$$

of a "model" variable $Z_{n}$ (for instance, a Poisson random variable) and an independent perturbation $Y_{n}$, when the model variable has "large" parameter. Our interest is in discrete random variables, and in cases where this simple-minded decomposition does not in fact exist. We have two motivations:

(1) In probabilistic number theory, it has been known since the proof by Rényi and Turán of the Erdős-Kac theorem that the random variable $\omega\left(N_{n}\right)$ given by the number of prime divisors (without multiplicity, for definiteness) of an integer $N_{n}$ uniformly chosen in the interval $\{1,2, \ldots, n\}$ has characteristic function given by

$$
\mathbb{E}\left\{e^{i \theta \omega\left(N_{n}\right)}\right\}=\mathbb{E}\left\{e^{i \theta Z_{n}}\right\} \Phi(\theta)(1+o(1))
$$

as $n \rightarrow \infty$, where $Z_{n} \sim \operatorname{Po}(\log \log n)$ is a Poisson variable with mean $\log \log n$ and $\Phi(\theta)$ is defined by

$$
\Phi(\theta)=\frac{1}{\Gamma\left(e^{i \theta}\right)} \prod_{p \text { prime }}\left(1+\frac{e^{i \theta}-1}{p}\right)\left(1-\frac{1}{p}\right)^{e^{i \theta}-1},
$$

the product being absolutely convergent for all $\theta$ real. This $\Phi(\theta)$ is not the characteristic function of a probability distribution, and hence formula (1.1) with $Z_{n} \sim \operatorname{Po}(\log \log n)$ cannot be true. However, we are nonetheless able to obtain explicit approximation statements for the law of $\omega\left(N_{n}\right)$ from the characteristic function identity.

2000 Mathematics Subject Classification. 62E17; 60F05, 60C05, 60E10, 11N60.

Key words and phrases. mod-Poisson convergence; characteristic function; Poisson-Charlier expansion; Erdős-Kac theorem.

EK supported in part by the National Science Foundation under agreement No. DMS-0635607 during a sabbatical stay at the Institute for Advanced Study.

ADB and AN supported in part by Schweizerischer Nationalfonds Projekte Nr. 20-117625/1 (ADB) and Nr. 200021_119970/1 (AN). 
Theorem 1.1. For every integer $r \geq 0$, there exist explicitly computable signed measures $\nu_{r, n}$ on the positive integers such that the total variation distance between the law of $\omega\left(N_{n}\right)$ and $\nu_{r, n}$ is of order $O\left\{(\log \log n)^{-(r+1) / 2}\right\}$ for $n \geq 3$.

This is proved, and formulas for the measures $\nu_{1, n}$ and $\nu_{2, n}$ are given, in Section 8.3. For more on the significance of the Rényi-Turán formula, comparison with the KeatingSnaith conjectures for the Riemann zeta function, and finite-field analogues, see Kowalski \& Nikeghbali (2009).

(2) In a beautiful paper, Hwang (1999) considered sequences of non-negative integer valued random variables $X_{n}$, whose probability generating functions $f_{X_{n}}$ satisfy

$$
e^{\lambda_{n}(1-z)} f_{X_{n}}(z) \rightarrow g(z)
$$

for all $z \in \mathbb{C}$ with $|z| \leq \eta$, for some $\eta>1$, where the function $g$ is analytic, and $\lim _{n \rightarrow \infty} \lambda_{n}=\infty$. This assumption is also intuitively related to a model (1.1). Under some extra conditions, Hwang exhibits bounds of order $O\left(\lambda_{n}^{-1}\right)$ on the accuracy of the approximation of the distribution of $X_{n}$ by a Poisson distribution with carefully chosen mean, close to $\lambda_{n}$. Hwang (1999) also notes that his methods can be applied to families of distributions other than the Poisson family, and gives examples using the Bessel family.

In this paper, we systematically consider sequences of integer valued random variables $X_{n}$, whose characteristic functions $\phi_{X_{n}}$ satisfy a condition which, in the Poisson context, is some strengthening of the convergence

$$
\exp \left\{\lambda_{n}\left(1-e^{i \theta}\right)\right\} \phi_{X_{n}}(\theta) \rightarrow \psi(\theta), \quad 0<|\theta| \leq \pi .
$$

Under suitable conditions, we derive explicit approximations to the distribution of $X_{n}$, in various metrics, by measures related to the Poisson model. The approximations can be made close to any given polynomial order in $\lambda_{n}^{-1 / 2}$, if the conditions are sharp enough and the measure is correspondingly chosen. The conditions that we require for these expansions are much weaker than those of Hwang (1999). For instance, his conditions require the $X_{n}$ to take only non-negative values, and to have exponential tails, neither of which conditions we need to impose.

Our basic result, Proposition 2.1, is very simple and explicit. It enables us to dispense with asymptotic settings, and to prove concrete error bounds. It also allows us to consider approximation by quite general families of distributions on the integers, instead of just the Poisson family, requiring only the replacement of the Poisson characteristic function in (1.2) by the characteristic function corresponding to the family chosen. This enables us to deduce expansions based on any discrete family of distributions, as shown in Section 4, without any extra effort. Indeed, the main problem would seem to be to identify the higher order terms in the expansions, but these turn out simply to be linear combinations of the higher order differences of the basic distribution: see (2.6).

In the Poisson context, the measures that result are the Poisson-Charlier measures. Our general results enable us to deduce a Poisson-Charlier approximation with error of order $O\left(\lambda_{n}^{-t / 2}\right)$, for any prescribed $t$, assuming that Hwang's conditions hold. We also show that the Poisson-Charlier expansions are valid under more general conditions, in which the $X_{n}$ may have only a few finite moments. The expansions are established in Section 5, and simpler, translated Poisson approximations are considered in Section 6 . We discuss some examples, to sums of independent integer valued random variables, to Hwang's setting and to our first motivation, proving Theorem 1.1, in Section 8. 


\section{The BASIC ESTIMATE}

The essence of our argument is the following elementary result, linking the closeness of finite signed measures $\mu$ and $\nu$ to the closeness of their characteristic functions, when these have a common factor involving a 'large' parameter $\rho$; for a finite signed measure $\zeta$ on $\mathbb{Z}$, the characteristic function $\phi_{\zeta}$ is defined by $\phi_{\zeta}(\theta):=\sum_{j \in \mathbb{Z}} e^{i j \theta} \zeta\{j\}$, for $|\theta| \leq \pi$.

Proposition 2.1. Let $\mu$ and $\nu$ be finite signed measures on $\mathbb{Z}$, with characteristic functions $\phi_{\mu}$ and $\phi_{\nu}$ respectively. Suppose that $\phi_{\mu}=\psi_{\mu} \chi$ and $\phi_{\nu}=\psi_{\nu} \chi$, where, for some $\gamma, \rho, t>0$

$$
\left|\psi_{\mu}(\theta)-\psi_{\nu}(\theta)\right| \leq \gamma|\theta|^{t} \quad \text { and } \quad|\chi(\theta)| \leq e^{-\rho \theta^{2}} \quad \text { for all } \quad|\theta| \leq \pi .
$$

Then there are explicit constants $\alpha_{1 t}$ and $\alpha_{2 t}$ such that

$$
\begin{aligned}
& \text { 1. } \sup _{j \in \mathbb{Z}}|\mu\{j\}-\nu\{j\}| \leq \alpha_{1 t} \gamma(\rho \vee 1)^{-(t+1) / 2} ; \\
& \text { 2. } \sup _{a \leq b \in \mathbb{Z}}|\mu\{[a, b]\}-\nu\{[a, b]\}| \leq \alpha_{2 t} \gamma(\rho \vee 1)^{-t / 2} .
\end{aligned}
$$

Proof. For any $j \in \mathbb{Z}$, the Fourier inversion formula gives

$$
\mu\{j\}-\nu\{j\}=\frac{1}{2 \pi} \int_{-\pi}^{\pi} e^{-i j \theta}\left(\psi_{\mu}(\theta)-\psi_{\nu}(\theta)\right) \chi(\theta) d \theta
$$

from which our assumptions imply directly that

$$
|\mu\{j\}-\nu\{j\}| \leq \frac{1}{2 \pi} \int_{-\pi}^{\pi} \gamma|\theta|^{t} \exp \left\{-\rho \theta^{2}\right\} d \theta .
$$

For $\rho \leq 1$, we thus have

$$
|\mu\{j\}-\nu\{j\}| \leq \frac{1}{2 \pi} \int_{-\pi}^{\pi} \gamma|\theta|^{t} d \theta \leq \frac{\pi^{t} \gamma}{t+1}=: \beta_{1 t} \gamma
$$

For $\rho \geq 1$, it is immediate that

$$
|\mu\{j\}-\nu\{j\}| \leq \frac{\gamma}{2 \pi}\left(\frac{1}{\sqrt{2 \rho}}\right)^{t+1} \int_{-\infty}^{\infty}|y|^{t} e^{-y^{2} / 2} d y \leq \beta_{1 t}^{\prime} \gamma \rho^{-(t+1) / 2},
$$

with $\beta_{1 t}^{\prime}:=2^{-(t+1) / 2} m_{t} / \sqrt{2 \pi}$; here, $m_{t}$ denotes the $t$-th absolute moment of the standard normal distribution. Setting

$$
\alpha_{1 t}:=\max \left\{\beta_{1 t}, \beta_{1 t}^{\prime}\right\}=\max \left\{2^{-(t+1) / 2} m_{t} / \sqrt{2 \pi}, \pi^{t} /(t+1)\right\},
$$

this proves part 1. The second part is similar, adding (2.2) over $a \leq j \leq b$, and estimating

$$
\frac{\left|e^{-i a \theta}-e^{-i(b+1) \theta}\right|}{\left|1-e^{-i \theta}\right|} \leq \frac{\pi}{|\theta|}, \quad|\theta| \leq \pi .
$$

This gives part 2, with

$$
\alpha_{2 t}:=\max \left\{2^{-t / 2} m_{t-1} \sqrt{\pi / 2}, \pi^{t} / t\right\}
$$

We shall principally be concerned with taking $\mu$ to be the distribution of a random variable $X$. We allow $\nu$ to be a signed measure, because in many cases, such as in the following canonical example and in the Poisson-Charlier expansions of Section 5, signed measures appear as the natural approximations. 
Let $X$ be an integer valued random variable with characteristic function $\phi_{X}:=\psi \chi$, where $\chi$ is the characteristic function of a (well known) probability distribution $R$ on $\mathbb{Z}$. Suppose that $\chi$ satisfies

$$
|\chi(\theta)| \leq e^{-\rho \theta^{2}}
$$

as for Proposition 2.1, and that $\psi$ can be approximated by a polynomial expansion around $\theta=0$ of the form

$$
\tilde{\psi}_{r}(\theta):=\sum_{l=0}^{r} \tilde{a}_{l}\left(e^{i \theta}-1\right)^{l},
$$

for real coefficients $\tilde{a}_{l}$ (and with $\tilde{a}_{0}=1$ ) and some $r \in \mathbb{N}_{0}$, in that

$$
\left|\psi(\theta)-\tilde{\psi}_{r}(\theta)\right| \leq K_{r \delta}|\theta|^{r+\delta}, \quad|\theta| \leq \pi,
$$

for some $0<\delta \leq 1$. Clearly, this is much like supposing that $\psi$ has a Taylor expansion around zero (in powers of $i \theta$ ), and hence that $X$ has a corresponding number of finite moments. However, the particular form chosen for the expansion has the advantage that the signed measure $\nu_{r}=\nu_{r}\left(R ; \tilde{a}_{1}, \ldots, \tilde{a}_{r}\right)$ having $\tilde{\psi}_{r} \chi$ as characteristic function can immediately be identified:

$$
\nu_{r}=\sum_{l=0}^{r}(-1)^{l} \tilde{a}_{l} D^{l} R,
$$

where the differences $D^{l} R$ of the probability measure $R$ are determined by iterating the relation $D R\{j\}:=R\{j\}-R\{j-1\}$. The following theorem now follows directly from Proposition 2.1.

Theorem 2.2. Let $X$ be a random variable on $\mathbb{Z}$ with distribution $P_{X}$. Suppose that its characteristic function $\phi_{X}$ is of the form $\psi \chi$, where $\chi$ is the characteristic function of a probability distribution $R$ and satisfies (2.3) above. Suppose also that (2.5) is satisfied, for some $r \in \mathbb{N}_{0}, \tilde{a}_{1}, \ldots, \tilde{a}_{r} \in \mathbb{R}$ and $\delta \geq 0$. Then, writing $t=r+\delta$, we have

$$
\begin{aligned}
& \text { 1. } \sup _{j \in \mathbb{Z}}\left|P_{X}\{j\}-\nu_{r}\{j\}\right| \leq \alpha_{1 t} K_{r \delta}(\rho \vee 1)^{-(t+1) / 2} ; \\
& \text { 2. } \sup _{a \leq b \in \mathbb{Z}}\left|P_{X}\{[a, b]\}-\nu_{r}\{[a, b]\}\right| \leq \alpha_{2 t} K_{r \delta}(\rho \vee 1)^{-t / 2},
\end{aligned}
$$

with $\alpha_{1 t}$ and $\alpha_{2 t}$ as in Proposition 2.1, and with $\nu_{r}=\nu_{r}\left(R ; \tilde{a}_{1}, \ldots, \tilde{a}_{r}\right)$ as defined in (2.6).

Remark. Note that Proposition 2.1 can be applied with $\psi_{\mu}=0$, corresponding to $\mu$ the zero measure, and $\psi_{\nu}(\theta)=\tilde{a}_{l}\left(e^{i \theta}-1\right)^{l}$, for any $1 \leq l \leq r$, showing that the contribution from the $l$-th term in the expansion to $\nu_{r}\{j\}$ is at most $\left|\tilde{a}_{l}\right| \alpha_{1 l}(\rho \vee 1)^{-(l+1) / 2}$, and that to $\nu_{r}\{[a, b]\}$ at most $\left|\tilde{a}_{l}\right| \alpha_{2 l}(\rho \vee 1)^{-l / 2}$. Thus, if $\rho$ is large and the coefficients $\tilde{a}_{l}$ moderate, the contributions decrease in powers of $\rho^{-1 / 2}$ as $l$ increases. In such circumstances, the signed measure $\nu_{r}$ can be seen as a perturbation of the underlying distribution $R$.

The simplest application of the above results arises when $\phi_{X}=\phi_{Y} p_{\lambda}$, where $p_{\lambda}(\theta)=$ $e^{\lambda\left(e^{i \theta}-1\right)}$ is the characteristic function of the Poisson distribution $\operatorname{Po}(\lambda)$ with mean $\lambda$, which satisfies (2.3) with $\rho=2 \pi^{-2} \lambda$, and $\phi_{Y}$ is the characteristic function associated with a random variable $Y$ on the integers. In this case, $X=Z+Y$ is the sum of two independent random variables, as in (1.1), with $Z \sim \operatorname{Po}(\lambda)$, and the situation is probabilistically very clear. For $w=w_{\theta}=e^{i \theta}-1$, we have $\phi_{Y}(\theta)=\mathbb{E}\left\{(1+w)^{Y}\right\}$. The latter expression has an expansion in powers of $w$ up to the term in $w^{r}$ if the $r$-th moment 
of $Y$ exists, with coefficients $\tilde{a}_{k}:=F_{k}(Y) / k !, 1 \leq k \leq r$, where $F_{k}(Y)$ denotes the $k$-th factorial moment of $Y$ :

$$
F_{k}(Y):=\sum_{l \geq k} \frac{l !}{(l-k) !} \mathbb{P}[Y=l]+\sum_{l \geq 1}(-1)^{k} \frac{(l+k-1) !}{(l-1) !} \mathbb{P}[Y=-l] .
$$

Thus the asymptotic expansion of $X$ around $\operatorname{Po}(\lambda)$ is simply derived from the factorial moments of the perturbing random variable $Y$, if they exist.

For example, we could take $\phi_{Y}$ to be the characteristic function of a random variable $Y_{s}$ with distribution

$$
\mathbb{P}\left[Y_{s}=-l\right]=s ! \frac{s}{l(l+1) \ldots(l+s)}, \quad l \geq 1,
$$

for some integer $s \geq 1$; the random variable has only $s-1$ moments, and takes negative values, so that Hwang's (1999) theorems cannot be applied. However, $Y_{s}$ has factorial moments

$$
F_{k}\left(Y_{s}\right)=(-1)^{k} s ! \sum_{l \geq 1} \frac{s}{(l+k) \ldots(l+s)}=(-1)^{k} k ! \frac{s}{s-k}, \quad 1 \leq k \leq s-1,
$$

and characteristic function

$$
\psi_{Y_{s}}(\theta)=1+\sum_{k=1}^{s-1}(-1)^{k} \frac{s}{s-k}\left(e^{i \theta}-1\right)^{k}-s\left(1-e^{i \theta}\right)^{s} \log \left(1-e^{-i \theta}\right),
$$

and (2.5) holds for $\tilde{\psi}_{r}$ as in (2.4), with $r=s-1$ and any $\delta<1$, for $\tilde{a}_{k}=F_{k}(Y) / k !=$ $(-1)^{k} s /(s-k)$. Hence, if $X=Z+Y_{s}$, where $Z \sim \operatorname{Po}(\lambda)$ is independent of $Y_{s}$, then Theorem 2.2 can be applied, approximating the distribution of $X$ by the signed measure $\nu_{s-1}\left(\operatorname{Po}(\lambda) ; \tilde{a}_{1}, \ldots, \tilde{a}_{s-1}\right)$.

\section{REFINEMENTS}

We frame our approximations in terms of three distances between (signed) measures $\mu$ and $\nu$ on the integers: the point metric

$$
d_{\mathrm{loc}}(\mu, \nu):=\sup _{j \in \mathbb{Z}}|\mu\{j\}-\nu\{j\}|,
$$

the Kolmogorov distance

$$
d_{\mathrm{K}}(\mu, \nu):=\sup _{j \in \mathbb{Z}}|\mu\{(-\infty, j]\}-\nu\{(-\infty, j]\}|,
$$

and the total variation norm

$$
\|\mu-\nu\|:=\sum_{j \in \mathbb{Z}}|\mu\{j\}-\nu\{j\}| .
$$

Other metrics could also be treated using our results. Proposition 2.1 immediately yields explicit bounds on $d_{\mathrm{loc}}(\mu, \nu)$ and $d_{\mathrm{K}}(\mu, \nu)$ in terms of the quantities specified in (2.1). However, for many applications, a slight weakening of its conditions is useful. The following result is proved in exactly the same way as Proposition 2.1.

Proposition 3.1. Let $\mu$ and $\nu$ be finite signed measures on $\mathbb{Z}$, with characteristic functions $\phi_{\mu}$ and $\phi_{\nu}$ respectively. Suppose that $\phi_{\mu}=\psi_{\mu} \chi$ and $\phi_{\nu}=\psi_{\nu} \chi$, where, for some 
$\theta_{0}, \varepsilon, \eta, \rho>0$ and for positive pairs $\gamma_{m}, t_{m}, 1 \leq m \leq M$, we have

$$
\begin{aligned}
& \left|\psi_{\mu}(\theta)-\psi_{\nu}(\theta)\right| \leq \sum_{m=1}^{M} \gamma_{m}|\theta|^{t_{m}}+\varepsilon \quad \text { and } \quad|\chi(\theta)| \leq e^{-\rho \theta^{2}}, \quad 0 \leq|\theta| \leq \theta_{0} \\
& \left|\phi_{\mu}(\theta)-\phi_{\nu}(\theta)\right| \leq \eta, \quad \theta_{0}<|\theta| \leq \pi
\end{aligned}
$$

Then, with notation as for Proposition 2.1, we have

$$
\begin{aligned}
& \text { 1. } \sup _{j \in \mathbb{Z}}|\mu\{j\}-\nu\{j\}| \leq \sum_{m=1}^{M} \gamma_{m} \alpha_{1 t_{m}}(\rho \vee 1)^{-\left(t_{m}+1\right) / 2}+\tilde{\alpha}_{1} \varepsilon+\tilde{\alpha}_{2} \eta ; \\
& \text { 2. } \quad \sup _{a_{0} \leq a \leq b \leq b_{0}}|\mu\{[a, b]\}-\nu\{[a, b]\}| \\
& \leq \sum_{m=1}^{M} \gamma_{m} \alpha_{2 t_{m}}(\rho \vee 1)^{-t_{m} / 2}+\left(b_{0}-a_{0}+1\right)\left(\tilde{\alpha}_{1} \varepsilon+\tilde{\alpha}_{2} \eta\right),
\end{aligned}
$$

where

$$
\tilde{\alpha}_{1}:=\left(\frac{\theta_{0}}{\pi} \wedge \frac{1}{2 \sqrt{\pi \rho}}\right) ; \quad \tilde{\alpha}_{2}:=\left(1-\frac{\theta_{0}}{\pi}\right)
$$

The presence of the factor $\left(b_{0}-a_{0}+1\right)$ in the second bound means that a direct bound on the Kolmogorov distance between the signed measures $\mu$ and $\nu$ is not immediately visible. The following corollary is however easily deduced; for a signed measure $\mu,|\mu|$ as usual denotes its variation.

Corollary 3.2. Under the conditions of Proposition 3.1,

$$
\begin{aligned}
& d_{\mathrm{K}}(\mu, \nu) \leq \inf _{a \leq b}\left(\varepsilon_{a b}^{(\mathrm{K})}+(|\mu|+|\nu|)\left\{[a, b]^{c}\right\}\right) ; \\
& \|\mu-\nu\| \leq \inf _{a \leq b}\left(\varepsilon_{a b}^{(1)}+(|\mu|+|\nu|)\left\{[a, b]^{c}\right\}\right),
\end{aligned}
$$

where

$$
\begin{aligned}
\varepsilon_{a b}^{(\mathrm{K})} & :=\sum_{m=1}^{M} \gamma_{m} \alpha_{2 t_{m}}(\rho \vee 1)^{-t_{m} / 2}+(b-a+1)\left(\tilde{\alpha}_{1} \varepsilon+\tilde{\alpha}_{2} \eta\right) \\
\varepsilon_{a b}^{(1)} & :=(b-a+1)\left\{\sum_{m=1}^{M} \gamma_{m} \alpha_{1 t_{m}}(\rho \vee 1)^{-\left(t_{m}+1\right) / 2}+\left(\tilde{\alpha}_{1} \varepsilon+\tilde{\alpha}_{2} \eta\right)\right\} .
\end{aligned}
$$

If also $\mu$ is a probability measure and $\nu(\mathbb{Z})=1$, then

$$
\begin{aligned}
& d_{\mathrm{K}}(\mu, \nu) \leq 2 \inf _{a \leq b}\left(\varepsilon_{a b}^{(\mathrm{K})}+|\nu|\left\{[a, b]^{c}\right\}\right) \\
& \|\mu-\nu\| \leq \inf _{a \leq b}\left(\varepsilon_{a b}^{(1)}+\varepsilon_{a b}^{(\mathrm{K})}+2|\nu|\left\{[a, b]^{c}\right\}\right) .
\end{aligned}
$$


Proof. The inequality for the total variation norm is immediate. For the Kolmogorov distance, by considering the possible positions of $x$ in relation to $a<b$, we have

$$
\begin{aligned}
& |\mu\{(-\infty, x]\}-\nu\{(-\infty, x]\}| \\
& \quad \leq \sup _{y<a}|\mu\{(-\infty, y]\}-\nu\{(-\infty, y]\}|+\sup _{a \leq y \leq b}|\mu\{[a, y]\}-\nu\{[a, y]\}| \\
& \quad+\sup _{y>b}|\mu\{(b, y]\}-\nu\{(b, y]\}| \\
& \quad \leq(|\mu|+|\nu|)\{(-\infty, a) \cup(b, \infty)\}+\varepsilon_{a b}^{(\mathrm{K})} .
\end{aligned}
$$

Remark. If $\mu$ is a probability measure and $\nu(\mathbb{Z})=1$, we have

$$
|\mu|\left\{[a, b]^{c}\right\}=1-\mu\{[a, b]\} \leq|1-\nu\{[a, b]\}|+\varepsilon_{a b}^{(\mathrm{K})} \leq|\nu|\left\{[a, b]^{c}\right\}+\varepsilon_{a b}^{(\mathrm{K})} .
$$

Under slightly stronger conditions than those of Proposition 2.1, a much neater total variation bound can be deduced; the argument is reminiscient of that in Presman (1983).

Proposition 3.3. Let $\mu$ and $\nu$ be finite signed measures on $\mathbb{Z}$, with characteristic functions $\phi_{\mu}=\psi_{\mu} \chi$ and $\phi_{\nu}=\psi_{\nu} \chi$ respectively, where $\chi(\theta):=e^{i \zeta \theta-u(\theta)}$ for some $\zeta \in \mathbb{R}$, and $u(0)=u^{\prime}(0)=0$. Suppose now that $u$ and the difference $d_{\mu \nu}:=\psi_{\mu}-\psi_{\nu}$ are both twice differentiable, that $d_{\mu \nu}^{\prime}(0)=0$ and that, for some $\gamma, \gamma^{\prime}>0, \rho \geq 1$ and $t \geq 2$,

$$
\left|d_{\mu \nu}^{\prime \prime}(\theta)\right| \leq \gamma|\theta|^{t-2}, \quad\left|u^{\prime \prime}(\theta)\right| \leq \gamma^{\prime} \rho \quad \text { and } \quad \Re\{u(\theta)\} \geq \rho \theta^{2} \text {, for all }|\theta| \leq \pi .
$$

Then there is a constant $\alpha^{\prime}:=\alpha^{\prime}\left(t, \gamma^{\prime}\right)$ such that

$$
\|\mu-\nu\| \leq \gamma \alpha^{\prime} \rho^{-t / 2} \text {. }
$$

Proof. First, the assumptions on $d_{\mu \nu}$ and $u$ give

$$
\begin{aligned}
\left|d_{\mu \nu}^{\prime}(\theta)\right| & \leq \frac{\gamma}{t-1}|\theta|^{t-1} ; \quad\left|d_{\mu \nu}(\theta)\right| \leq \frac{\gamma}{t(t-1)}|\theta|^{t} ; \\
\left|u^{\prime}(\theta)\right| & \leq \gamma^{\prime} \rho|\theta| .
\end{aligned}
$$

In particular, for $|j-\zeta|<1+\lceil\sqrt{\rho}\rceil$, we can apply part 1 of Proposition 2.1, which gives

$$
|\mu\{j\}-\nu\{j\}| \leq \frac{\alpha_{1 t} \gamma}{t(t-1)} \rho^{-(t+1) / 2} .
$$

For the remaining $j$, integrating the Fourier inversion formula (2.2) twice by parts gives

$$
\begin{aligned}
\mu\{j\}-\nu\{j\}=-\frac{1}{2 \pi(j-\zeta)^{2}} \int_{-\pi}^{\pi} e^{-i(j-\zeta) \theta} & \left(d_{\mu \nu}^{\prime \prime}(\theta)-2 d_{\mu \nu}^{\prime}(\theta) u^{\prime}(\theta)\right. \\
& \left.+d_{\mu \nu}(\theta)\left\{\left(u^{\prime}(\theta)\right)^{2}-u^{\prime \prime}(\theta)\right\}\right) e^{-u(\theta)} d \theta .
\end{aligned}
$$

Substituting the bounds from (3.1) into (3.3) gives

$$
\begin{aligned}
& |\mu\{j\}-\nu\{j\}| \\
& \quad \leq \frac{1}{2 \pi(j-\zeta)^{2}} \int_{-\pi}^{\pi} \gamma\left\{|\theta|^{t-2}+\frac{2 \gamma^{\prime} \rho}{t-1}|\theta|^{t}+\frac{\gamma^{\prime} \rho}{t(t-1)}|\theta|^{t}\left(1+\gamma^{\prime} \rho \theta^{2}\right)\right\} e^{-\rho \theta^{2}} d \theta \\
& \quad \leq \frac{1}{(j-\zeta)^{2}} \gamma \beta^{\prime}\left(t, \gamma^{\prime}\right) \rho^{-(t-1) / 2},
\end{aligned}
$$


after some calculation, where, with $m_{t}$ as in Proposition 2.1,

$$
\beta^{\prime}\left(t, \gamma^{\prime}\right):=\frac{m_{t-2}}{4 t 2^{t / 2} \sqrt{\pi}}\left\{4 t+2(2 t+1) \gamma^{\prime}+(t+1)\left(\gamma^{\prime}\right)^{2}\right\}
$$

Hence

$$
\sum_{|j-\zeta| \geq 1+\lceil\sqrt{\rho}\rceil}|\mu\{j\}-\nu\{j\}| \leq 2 \gamma \beta^{\prime}\left(t, \gamma^{\prime}\right) \rho^{-t / 2}
$$

and the proposition follows directly, with $\alpha^{\prime}\left(t, \gamma^{\prime}\right):=2 \beta^{\prime}\left(t, \gamma^{\prime}\right)+\frac{6 \alpha_{1 t}}{t(t-1)}$.

Note that, in the Poisson context, with $u(\theta)=\lambda\left(1-e^{i \theta}+i \theta\right)$, we take $\rho=2 \lambda / \pi^{2}$ as before, and then $\gamma^{\prime}=\pi^{2} / 2$. Note also that, if $d_{\mu \nu}(\theta)=\hat{d}_{\mu \nu}\left(e^{i \theta}-1\right)$, with $\hat{d}_{\mu \nu}(0)=$ $\hat{d}_{\mu \nu}^{\prime}(0)=0$ and $\left|\hat{d}_{\mu \nu}^{\prime \prime}(w)\right| \leq \hat{\gamma}|w|^{t-2}$ in $|w| \leq 2$, then $d_{\mu \nu}(0)=d_{\mu \nu}^{\prime}(0)=0$ and

$$
\left|d_{\mu \nu}^{\prime \prime}(\theta)\right| \leq\left(1+\frac{2}{t-1}\right) \hat{\gamma}|\theta|^{t-2}
$$

in $|\theta| \leq \pi$.

The above proposition, like Proposition 2.1, has conditions that are often satisfied almost, but not quite, in applications. The next result allows the sort of freedom envisaged in Proposition 3.1. It is useful when $\rho$ is large, the quantities $\varepsilon$ and $\eta$ are, for instance, exponentially small with $\rho$, and the same is true for $|\nu|\left\{[-\rho, \rho]^{c}\right\}$. In such circumstances, it yields a bound of (slightly) sharper order than can be deduced from Corollary 3.2, since there it is necessary to choose $a$ and $b$ in such a way that $\rho^{-1 / 2}(b-a)$ is large, if $|\nu|\left\{[a, b]^{c}\right\}$ is to be made small, and the factor $\rho^{-1 / 2}(b-a)$ then multiplies the main element of the bound thus obtained.

Proposition 3.4. Let $\mu$ and $\nu$ be finite signed measures on $\mathbb{Z}$, with characteristic functions $\phi_{\mu}=\psi_{\mu} \chi$ and $\phi_{\nu}=\psi_{\nu} \chi$ respectively, where $\chi(\theta):=e^{i \zeta \theta-u(\theta)}$ for some $\zeta \in \mathbb{R}$, and for some function $u$ satisfying the same conditions as in Proposition 3.3. Suppose that $\tilde{d}_{\mu \nu}$ is a twice differentiable function such that $\tilde{d}_{\mu \nu}(0)=\tilde{d}_{\mu \nu}^{\prime}(0)=0$ and that

$$
\left|\tilde{d}_{\mu \nu}^{\prime \prime}(\theta)\right| \leq \sum_{m=1}^{M} \gamma_{m}|\theta|^{t_{m}-2}
$$

for some $t_{m} \geq 2$ and $\gamma_{m}>0,1 \leq m \leq M$; assume that

$$
\begin{aligned}
e^{-\rho \theta^{2}}\left|\psi_{\mu}(\theta)-\psi_{\nu}(\theta)-\tilde{d}_{\mu \nu}(\theta)\right| & \leq \varepsilon, & & |\theta| \leq \theta_{0} ; \\
\left|\phi_{\mu}(\theta)-\phi_{\nu}(\theta)\right| & \leq \eta, & & \theta_{0}<|\theta| \leq \pi,
\end{aligned}
$$

for some $\varepsilon, \eta>0$ and for some $\theta_{0}$ with $\theta_{0} \leq \pi / 4$ and $\rho \theta_{0}^{2} \geq \log \rho \geq 0$. Then there is a function $\alpha^{\prime}:=\alpha^{\prime}(t, \gamma)$ such that, with $\gamma^{\prime}$ as in Proposition 3.3,

$$
\|\mu-\nu\| \leq \sum_{m=1}^{M} \gamma_{m} \alpha^{\prime}\left(t_{m}, \gamma^{\prime}\right) \rho^{-t_{m} / 2}+3 \rho \max \{\varepsilon, \eta\}+(|\mu|+|\nu|)\left\{[-\rho, \rho]^{c}\right\} .
$$

If $\mu$ is a probability measure and $\nu(\mathbb{Z})=1$, then

$$
\|\mu-\nu\| \leq 2 \sum_{m=1}^{M} \gamma_{m} \alpha^{\prime}\left(t_{m}, \gamma^{\prime}\right) \rho^{-t_{m} / 2}+6 \rho \max \{\varepsilon, \eta\}+2|\nu|\left\{[-\rho, \rho]^{c}\right\} .
$$


Proof. We begin by observing that a function $w:(-\infty, 0] \rightarrow \mathbb{R}$ with $w(0)=a$ and $w^{\prime}(0)=$ $b$ can be continued differentiably on $[0, \infty)$ by a piecewise quadratic function such that $\left|w^{\prime \prime}(x)\right| \leq c$ for all $x>0$ for which $w^{\prime \prime}(x)$ is defined, and such that $w(x)=0$ for all

$$
x \geq \frac{1}{c}\left\{|b|+2 \sqrt{\left|a c+\frac{1}{2} \operatorname{sgn}(b) b^{2}\right|}\right\},
$$

with $\max _{x \geq 0}|w(x)| \leq|a|+b^{2} / 2 c$. We use this to continue the real and imaginary parts of $\tilde{w}(\theta):=e^{-u(\theta)} \tilde{d}_{\mu \nu}(\theta)$ into $\theta_{0} \leq|\theta| \leq \pi$ in such a way that $\tilde{w}$ is piecewise twice differentiable and satisfies

$$
\tilde{w}(-\pi)=\tilde{w}(\pi)=\tilde{w}^{\prime}(-\pi)=\tilde{w}^{\prime}(\pi)=0,
$$

with the second derivatives of the real and imaginary parts suitably bounded. We do so by treating $\tilde{w}$ as a sum of $M$ pieces, reflecting the condition (3.6); for the $m$ 'th piece $\tilde{w}_{m}$, defining $\kappa_{1}(t, \gamma):=(t+\gamma) /\{t(t-1)\}$, we have

$$
\begin{aligned}
\left|\tilde{w}_{m}\left(\theta_{0}\right)\right| & \leq \frac{\gamma_{m}}{t_{m}\left(t_{m}-1\right)} \theta_{0}^{t_{m}} e^{-\rho \theta_{0}^{2}} \\
& \leq\left|a_{m}\right|:=t_{m}^{-1} \gamma_{m} \kappa_{1}\left(t_{m}, \gamma^{\prime}\right) \theta_{0}^{t_{m}} e^{-\rho \theta_{0}^{2}} \\
\left|\tilde{w}_{m}^{\prime}\left(\theta_{0}\right)\right| & \leq \frac{\gamma_{m}}{t_{m}\left(t_{m}-1\right)} \theta_{0}^{t_{m}-1} e^{-\rho \theta_{0}^{2}}\left\{t_{m}+\gamma^{\prime} \rho \theta_{0}^{2}\right\} \\
& \leq\left|b_{m}\right|:=\gamma_{m} \kappa_{1}\left(t_{m}, \gamma^{\prime}\right) \rho \theta_{0}^{t_{m}+1} e^{-\rho \theta_{0}^{2}}
\end{aligned}
$$

with the same bounds holding also at $-\theta_{0}$, and we restrict the second derivatives of its continuation by taking

$$
c_{m}:=4 \gamma_{m} \kappa_{1}\left(t_{m}, \gamma^{\prime}\right) \rho^{2} \theta_{0}^{t_{m}+2} e^{-\rho \theta_{0}^{2}}
$$

in $\theta_{0} \leq|\theta| \leq \pi$. The length of the $\theta$-interval beyond $\theta_{0}$ on which $\tilde{w}_{m}$ is not identically zero is thus bounded by

$$
\frac{1}{c_{m}}\left\{\left|b_{m}\right|(1+\sqrt{2})+2 \sqrt{\left|a_{m}\right| c_{m}}\right\} \leq \frac{1+3 \sqrt{2}}{4 \rho \theta_{0}} \leq h:=\frac{2}{\rho \theta_{0}},
$$

from (3.7) and (3.8), the bound being the same for all $m$; note that

$$
h \leq \frac{2 \theta_{0}}{\rho \theta_{0}^{2}} \leq \frac{\pi}{2}
$$

since $\theta_{0} \leq \pi / 4$ and $\rho \theta_{0}^{2} \geq 1$. From this and (3.9), it follows also that

$$
\int_{\theta_{0} \leq|\theta| \leq \pi}\left|\tilde{w}_{m}^{\prime \prime}(\theta)\right| d \theta \leq 4 h c_{m} \leq 32 \gamma_{m} \kappa_{1}\left(t_{m}, \gamma^{\prime}\right) \rho \theta_{0}^{t_{m}+1} e^{-\rho \theta_{0}^{2}},
$$

and, using (3.7) and (3.8), that

$$
\rho \int_{\theta_{0} \leq|\theta| \leq \pi}\left|\tilde{w}_{m}(\theta)\right| d \theta \leq 4 h \rho\left\{\left|a_{m}\right|+b_{m}^{2} / 2 c_{m}\right\} \leq 5 \gamma_{m} \kappa_{1}\left(t_{m}, \gamma^{\prime}\right) \theta_{0}^{t_{m}-1} e^{-\rho \theta_{0}^{2}} .
$$

The proof now follows the lines of those of Propositions 3.1 and 3.3. We begin by writing

$$
\begin{aligned}
& \mu\{j\}-\nu\{j\}=\frac{1}{2 \pi} \int_{-\pi}^{\pi} e^{-i j \theta}\left(\phi_{\mu}(\theta)-\phi_{\nu}(\theta)\right) d \theta \\
& \leq \frac{1}{2 \pi} \int_{-\pi}^{\pi} e^{-i(j-\zeta) \theta}\left\{e^{-u(\theta)}\left[\psi_{\mu}(\theta)-\psi_{\nu}(\theta)-\tilde{d}_{\mu \nu}(\theta)\right]+\tilde{w}(\theta)\right\} d \theta .
\end{aligned}
$$

Now, by assumption,

$$
\left|e^{-u(\theta)}\left[\psi_{\mu}(\theta)-\psi_{\nu}(\theta)-\tilde{d}_{\mu \nu}(\theta)\right]\right| \leq \varepsilon
$$


in $|\theta| \leq \theta_{0}$, whereas, in $\theta_{0}<|\theta| \leq \pi$, it is bounded by $\eta+|\tilde{w}(\theta)|$. Hence, for any $j$, we use (3.12) to give

$$
\begin{aligned}
& \left|\frac{1}{2 \pi} \int_{-\pi}^{\pi} e^{-i(j-\zeta) \theta} e^{-u(\theta)}\left[\psi_{\mu}(\theta)-\psi_{\nu}(\theta)-\tilde{d}_{\mu \nu}(\theta)\right] d \theta\right| \\
& \quad \leq \max \{\varepsilon, \eta\}+\frac{5}{2 \pi \rho} \sum_{m=1}^{M} \gamma_{m} \kappa_{1}\left(t_{m}, \gamma^{\prime}\right) \theta_{0}^{t_{m}-1} e^{-\rho \theta_{0}^{2}}
\end{aligned}
$$

Noting also that, if $\rho \theta^{2} \geq \log \rho \geq 0$ and $\theta>0$, then

$$
\rho^{t / 2} \theta^{t-1} e^{-\rho \theta^{2}}=\rho^{1 / 2}\left(\rho \theta^{2}\right)^{(t-1) / 2} e^{-\rho \theta^{2}} \leq k_{2}(t),
$$

for $k_{2}(t)=\{(t-1) / e\}^{(t-1) / 2} \max \left\{1,2^{-(t-1) / 2} \sqrt{t-1}\right\}$, it follows that

$$
\begin{aligned}
& \left|\frac{1}{2 \pi} \int_{-\pi}^{\pi} e^{-i(j-\zeta) \theta} e^{-u(\theta)}\left[\psi_{\mu}(\theta)-\psi_{\nu}(\theta)-\tilde{d}_{\mu \nu}(\theta)\right] d \theta\right| \\
& \quad \leq \max \{\varepsilon, \eta\}+\frac{5}{2 \pi \rho} \sum_{m=1}^{M} \gamma_{m} \kappa_{1}\left(t_{m}, \gamma^{\prime}\right) k_{2}\left(t_{m}\right) \rho^{-t_{m} / 2}
\end{aligned}
$$

This bounds the first element of (3.13).

For the second element of (3.13), we begin by considering values of $j$ such that $|j-\zeta|<$ $1+\lceil\sqrt{\rho}\rceil$. Here, we write

$$
\frac{1}{2 \pi} \int_{-\pi}^{\pi}|\tilde{w}(\theta)| d \theta \leq \frac{1}{2 \pi} \int_{|\theta| \leq \theta_{0}} e^{-\rho \theta^{2}}\left|\tilde{d}_{\mu \nu}(\theta)\right| d \theta+\frac{1}{2 \pi} \sum_{m=1}^{M} \int_{\theta_{0}<|\theta| \leq \pi}\left|\tilde{w}_{m}(\theta)\right| d \theta .
$$

The first integral is bounded, as in the proof of Propositions 2.1 and 3.1, by

$$
\sum_{m=1}^{M} \gamma_{m} \frac{\alpha_{1 t_{m}}}{t_{m}\left(t_{m}-1\right)} \rho^{-\left(t_{m}+1\right) / 2}
$$

and the second, as above, by

$$
\frac{5}{2 \pi \rho} \sum_{m=1}^{M} \gamma_{m} \kappa_{1}\left(t_{m}, \gamma^{\prime}\right) k_{2}\left(t_{m}\right) \rho^{-t_{m} / 2}
$$

giving the bound

$$
\begin{aligned}
& \left|\frac{1}{2 \pi} \int_{-\pi}^{\pi} e^{-i(j-\zeta) \theta} \tilde{w}(\theta) d \theta\right| \\
& \quad \leq \sum_{m=1}^{M} \gamma_{m}\left\{\frac{\alpha_{1 t_{m}}}{t_{m}\left(t_{m}-1\right)}+\frac{5}{2 \pi} \kappa_{1}\left(t_{m}, \gamma^{\prime}\right) k_{2}\left(t_{m}\right)\right\} \rho^{-\left(t_{m}+1\right) / 2},
\end{aligned}
$$

since $\rho \geq 1$. The interval containing such $j$ values contains at most $5+2 \sqrt{\rho}$ integers.

For $|j-\zeta| \geq 1+\lceil\sqrt{\rho}\rceil$, integrating twice by parts, it follows that

$$
\frac{1}{2 \pi} \int_{-\pi}^{\pi} e^{-i(j-\zeta) \theta} \tilde{w}(\theta) d \theta=-\frac{1}{2 \pi(j-\zeta)^{2}} \int_{-\pi}^{\pi} e^{-i(j-\zeta) \theta} \tilde{w}^{\prime \prime}(\theta) d \theta
$$


The right hand side can be bounded as in (3.4), but with an additional element of

$$
\begin{aligned}
& \frac{1}{2 \pi(j-\zeta)^{2}} \sum_{m=1}^{M} \int_{\theta_{0}<|\theta| \leq \pi}\left|\tilde{w}_{m}^{\prime \prime}(\theta)\right| d \theta \\
& \leq \frac{16}{\pi(j-\zeta)^{2}} \sum_{m=1}^{M} \gamma_{m} \kappa_{1}\left(t_{m}, \gamma^{\prime}\right) \rho \theta_{0}^{t_{m}+1} e^{-\rho \theta_{0}^{2}} \\
& \leq \frac{16}{\pi(j-\zeta)^{2}} \sum_{m=1}^{M} \gamma_{m} \kappa_{1}\left(t_{m}, \gamma^{\prime}\right) k_{3}\left(t_{m}\right) \rho^{-\left(t_{m}-1\right) / 2}
\end{aligned}
$$

from $(3.11)$, with $k_{3}(t):=\{(t+1) / 2 e\}^{(t+1) / 2}$. As a result, we find that, for $|j-\zeta| \geq$ $1+\lceil\sqrt{\rho}\rceil$, the second element of $(3.13)$ can be bounded by

$$
\begin{aligned}
& \left|\frac{1}{2 \pi} \int_{-\pi}^{\pi} e^{-i(j-\zeta) \theta} \tilde{w}(\theta) d \theta\right| \\
& \quad \leq \frac{1}{(j-\zeta)^{2}} \sum_{m=1}^{M} \gamma_{m}\left\{\beta^{\prime}\left(t_{m}, \gamma^{\prime}\right)+\frac{16}{\pi} \kappa_{1}\left(t_{m}, \gamma^{\prime}\right) k_{3}\left(t_{m}\right)\right\} \rho^{-\left(t_{m}-1\right) / 2} .
\end{aligned}
$$

The final step is to write

$$
\|\mu-\nu\| \leq \sum_{|j| \leq \rho}|\mu\{j\}-\nu\{j\}|+(|\mu|+|\nu|)\left\{[-\rho, \rho]^{c}\right\}
$$

and to note that, if $\mu$ is a probability measure and $\nu(\mathbb{Z})=1$, then

$$
\begin{aligned}
|\mu|\left\{[a, b]^{c}\right\} & =1-\mu\{[a, b]\} \leq|1-\nu\{[a, b]\}|+|\nu\{[a, b]\}-\mu\{[a, b]\}| \\
& \leq|\nu|\left\{[a, b]^{c}\right\}+\sum_{j=a}^{b}|\mu\{j\}-\nu\{j\}| .
\end{aligned}
$$

Remark. The interval $[-\rho, \rho]$ could be replaced by an interval $[-k \rho, k \rho]$, for any fixed $k \geq 1$, in the statement of the theorem, at the expense of multiplying the remaining bounds by the factor $k$.

\section{Applying the BOUndS}

Let $R_{\lambda}, \lambda \geq 0$, be a family of probability distributions on the integers. Assume that, for $Z_{\lambda} \sim R_{\lambda}, \mu(\lambda):=\mathbb{E} Z_{\lambda}$ and $\sigma^{2}(\lambda):=\operatorname{Var} Z_{\lambda}$ exist, and are both uniformly continuous functions of $\lambda$, with $\sigma^{2}(\lambda)$ strictly increasing from zero to infinity with $\lambda$. Suppose also that the characteristic function $r_{\lambda}$ of $R_{\lambda}$ can be written in the form $r_{\lambda}(\theta)=$ $\exp \left\{i \zeta_{\lambda} \theta-u_{\lambda}(\theta)\right\}$, with $u_{\lambda}^{\prime}(0)=0$, and that

$$
h(\lambda):=\inf _{0<|\theta| \leq \pi}\left(-\theta^{-2} \Re\left\{u_{\lambda}(\theta)\right\}\right)>0 .
$$

Then $\left|r_{\lambda}(\theta)\right| \leq e^{-h(\lambda) \theta^{2}}$ for $0 \leq|\theta| \leq \pi$, and so $R_{\lambda}$ can play the role of $R$ in Theorem 2.2, with $h(\lambda)$ for $\rho$. This leads to the following result.

Corollary 4.1. Let $X$ be an integer valued random variable with distribution $P_{X}$ and characteristic function $\phi_{X}:=\psi r_{\lambda}$, where $r_{\lambda}$ is as above and $h(\lambda) \geq 1$. Then, if (2.5) is satisfied for $\tilde{\psi}_{r}$, as defined in (2.4), for some $r \in \mathbb{N}_{0}, \tilde{a}_{1}, \ldots, \tilde{a}_{r} \in \mathbb{R}$ and $\delta \geq 0$, then, 
writing $t=r+\delta$, we have

$$
\begin{aligned}
& \text { 1. } \sup _{j \in \mathbb{Z}}\left|P_{X}\{j\}-\nu_{r}\{j\}\right| \leq \alpha_{1 t} K_{r \delta} h(\lambda)^{-(t+1) / 2} ; \\
& \text { 2. } \sup _{a \leq b \in \mathbb{Z}}\left|P_{X}\{[a, b]\}-\nu_{r}\{[a, b]\}\right| \leq \alpha_{2 t} K_{r \delta} h(\lambda)^{-t / 2},
\end{aligned}
$$

with $\alpha_{1 t}$ and $\alpha_{2 t}$ as in Proposition 2.1, and with $\nu_{r}=\nu_{r}\left(R_{\lambda} ; \tilde{a}_{1}, \ldots, \tilde{a}_{r}\right)$ as defined in (2.6). Furthermore, if $r \geq 2$ and $\psi-\tilde{\psi}_{r}$ and $u_{\lambda}$ are both twice differentiable, and if

$$
\left|\psi^{\prime \prime}(\theta)-\tilde{\psi}_{r}^{\prime \prime}(\theta)\right| \leq \widetilde{K}_{r \delta}|\theta|^{t-2} \text { and }\left|u_{\lambda}^{\prime \prime}(\theta)\right| \leq \gamma^{\prime} h(\lambda) \quad \text { for all }|\theta| \leq \pi,
$$

for constants $\widetilde{K}_{r \delta}$ and $\gamma^{\prime}$, then

$$
\text { 3. }\left\|P_{X}-\nu_{r}\right\| \leq \alpha^{\prime} \widetilde{K}_{r \delta} h(\lambda)^{-t / 2}
$$

for $\alpha^{\prime}:=\alpha^{\prime}\left(t, \gamma^{\prime}\right)$ as in Proposition 3.3.

Remark. Taking $\psi_{\mu}=0$ and $\psi_{\nu}=\left(e^{i \theta}-1\right)^{l}$ in Proposition 3.3 for $l \geq 2$ gives $\left|d_{\mu \nu}^{\prime \prime}(\theta)\right| \leq l(l+1)|\theta|^{l-2}$ and $d_{\mu \nu}^{\prime}(0)=0$. Hence the contribution from the $l$-th term in the signed measure $\nu_{r}$ of $(2.6)$ has total variation norm at most $\alpha^{\prime} l(l+1)\left|\tilde{a}_{l}\right| h(\lambda)^{-l / 2}$, for $2 \leq l \leq r$.

The use of signed measures to approximate probability distributions is convenient, but not very natural. However, the signed measures $\nu_{1}\left(R_{\lambda} ; \tilde{a}_{1}\right)$ and $\nu_{2}\left(R_{\lambda} ; \tilde{a}_{1}, \tilde{a}_{2}\right)$ can be replaced by suitably translated members of the family $\left\{R_{\lambda}, \lambda>0\right\}$, with the same asymptotic rate of approximation, by fitting the first two moments, a procedure analogous to that used in the Berry-Esseen theorem. The signed measure $\nu_{2}$ has mean and variance given by

$$
\mu_{*}=\mu(\lambda)+\tilde{a}_{1} ; \quad \sigma_{*}^{2}=\sigma^{2}(\lambda)+2 \tilde{a}_{2}+\tilde{a}_{1}\left(1-\tilde{a}_{1}\right)
$$

and the corresponding equations for $\nu_{1}$ just have $\tilde{a}_{2}=0$. However, when matching these moments, only integer translations $m$ of $R_{\lambda}$ can be allowed, since the distributions must remain on the integers, and so it is not possible to match both moments exactly within the family. To circumvent this, we extend to approximation by a member of the family of probability distributions $Q_{m p}\left(R_{\lambda^{\prime}}\right)$, for $\lambda^{\prime}>0, m \in \mathbb{Z}$ and $0 \leq p<1$, where

$$
Q_{m p}\left(R_{\lambda^{\prime}}\right)\{j\}:=p R_{\lambda^{\prime}}\{j-m-1\}+(1-p) R_{\lambda^{\prime}}\{j-m\} .
$$

If $Z \sim R_{\lambda^{\prime}}$, then $Q_{m p}\left(R_{\lambda^{\prime}}\right)$ is the distribution of $Z+m+I$, where $I \sim \operatorname{Be}(p)$ is independent of $Z$. $Q_{m p}\left(R_{\lambda^{\prime}}\right)$ has characteristic function $q_{m p}\left(R_{\lambda^{\prime}}\right)$ given by

$$
q_{m p}\left(R_{\lambda^{\prime}}\right)(\theta):=e^{i m \theta}\left(1+p\left(e^{i \theta}-1\right)\right) r_{\lambda^{\prime}}(\theta),
$$

similar to the measure $\nu_{2}\left\{R_{\lambda^{\prime}} ; m+p,\left(\begin{array}{c}m \\ 2\end{array}\right)+m p\right\}$, but with terms of higher order as powers of $\left(e^{i \theta}-1\right)$ as well.

Within the family $\left\{Q_{m p}\left(R_{\lambda^{\prime}}\right) ; \lambda^{\prime}>0, m \in \mathbb{Z}, 0 \leq p<1\right\}$, we can always find a member having a given mean $\mu_{*}$ and variance $\sigma_{*}^{2}$, provided that $\sigma_{*}^{2} \geq 1 / 4$, by solving the equations

$$
\mu_{*}=\mu\left(\lambda^{\prime}\right)+m+p ; \quad \sigma_{*}^{2}=\sigma^{2}\left(\lambda^{\prime}\right)+p(1-p) .
$$

To do so, let $\lambda_{p}$ solve $\sigma^{2}\left(\lambda_{p}\right)=\sigma_{*}^{2}-p(1-p)$, possible for $0 \leq p \leq 1$, since $\sigma_{*}^{2} \geq 1 / 4$ and the function $\sigma^{2}$ has an inverse; note also that $\lambda_{0}=\lambda_{1}$. Define $m_{p}:=\mu_{*}-\mu\left(\lambda_{p}\right)-p$, continuous under the assumptions on $\mu$, and observe that $m_{0}=m_{1}+1$. Hence the value $m=\left\lfloor m_{0}\right\rfloor$ is realized in the form $m_{p}$ for some $0 \leq p<1$, and then $\lambda_{p}, m_{p}$ and $p$ satisfy (4.24). 
We now show that $Q_{m p}\left(R_{\lambda^{\prime}}\right)$ and $\nu_{r}=\nu_{r}\left(R_{\lambda} ; \tilde{a}_{1}, \ldots, \tilde{a}_{r}\right)$ are close to order $O\{(h(\lambda) \vee$ $\left.1)^{-3 / 2}\right\}$, under some assumptions on the family $R_{\lambda}$. We assume that $\mu$ and the inverse function $\left(\sigma^{2}\right)^{-1}$ are both uniformly continuous, and that, for any $\lambda, \lambda^{\prime}>0$,

$$
d_{\lambda, \lambda^{\prime}}(\theta):=\frac{r_{\lambda^{\prime}}(\theta)}{r_{\lambda}(\theta)}-\left\{1+b_{1}\left(e^{i \theta}-1\right)+b_{2}\left(e^{i \theta}-1\right)^{2}\right\}
$$

satisfies

$$
d_{\lambda, \lambda^{\prime}}(0)=d_{\lambda, \lambda^{\prime}}^{\prime}(0)=0 ; \quad\left|d_{\lambda, \lambda^{\prime}}^{\prime \prime}(\theta)\right| \leq \gamma\left(\left|\lambda-\lambda^{\prime}\right|\right)|\theta| \text { in }|\theta| \leq \pi
$$

where, from moment considerations,

$$
\begin{aligned}
& b_{1}:=b_{1}\left(\lambda, \lambda^{\prime}\right):=\mu\left(\lambda^{\prime}\right)-\mu(\lambda) \\
& b_{2}:=b_{2}\left(\lambda, \lambda^{\prime}\right):=\frac{1}{2}\left\{\sigma^{2}\left(\lambda^{\prime}\right)-\sigma^{2}(\lambda)-b_{1}\left(1-b_{1}\right)\right\} .
\end{aligned}
$$

If this is the case, then the choices of $\lambda^{\prime}, m, p$ in (4.24) when $\mu_{*}$ and $\sigma_{*}^{2}$ are given by (4.21) are such as to give

$$
\tilde{a}_{1}=b_{1}+m+p ; \quad \tilde{a}_{2}=b_{2}+(m+p) b_{1}+m p+\left(\begin{array}{c}
m \\
2
\end{array}\right),
$$

so that $Q_{m p}\left(R_{\lambda^{\prime}}\right)$ and $\nu_{r}=\nu_{r}\left(R_{\lambda} ; \tilde{a}_{1}, \ldots, \tilde{a}_{r}\right)$ have the same mean and variance; what is more, $m,\left|\lambda^{\prime}-\lambda\right|,\left|\mu\left(\lambda^{\prime}\right)-\mu(\lambda)\right|$ and $\left|\sigma^{2}\left(\lambda^{\prime}\right)-\sigma^{2}(\lambda)\right|$ are uniformly bounded for $\left(\tilde{a}_{1}, \tilde{a}_{2}\right)$ in any compact set. It thus follows, with this choice of $\lambda^{\prime}, m, p$, that $q_{m p}\left(R_{\lambda^{\prime}}\right)(\theta)$ can be written as $r_{\lambda}(\theta) \psi_{\lambda, \lambda^{\prime}}(\theta)$, with

$$
\psi_{\lambda, \lambda^{\prime}}(\theta)=\left\{d_{\lambda, \lambda^{\prime}}(\theta)+\left[1+b_{1}\left(e^{i \theta}-1\right)+b_{2}\left(e^{i \theta}-1\right)^{2}\right]\right\} e^{i \theta m}\left(1+p\left(e^{i \theta}-1\right)\right),
$$

and with

$$
\hat{d}(\theta):=\left[1+b_{1}\left(e^{i \theta}-1\right)+b_{2}\left(e^{i \theta}-1\right)^{2}\right] e^{i \theta m}\left(1+p\left(e^{i \theta}-1\right)\right)-\tilde{\psi}_{r}(\theta)
$$

satisfying

$$
\hat{d}(0)=\hat{d}^{\prime}(0)=0 ; \quad\left|\hat{d}^{\prime \prime}(\theta)\right| \leq \hat{\gamma}|\theta|, \text { in }|\theta| \leq \pi,
$$

where $\hat{\gamma}=\hat{\gamma}\left(\tilde{a}_{1}, \ldots, \tilde{a}_{r}\right)$ remains bounded if $\tilde{a}_{1}, \ldots, \tilde{a}_{r}$ do. In view of $(4.25)-(4.27)$, the difference $\psi_{\lambda, \lambda^{\prime}}-\tilde{\psi}_{r}$ satisfies $(4.20)$ for a constant $\widetilde{K}_{r \delta}=\widetilde{K}_{r \delta}\left(\tilde{a}_{1}, \ldots, \tilde{a}_{r}\right)$, whose definition depends on the family $R_{\lambda}$, and hence, from Corollary 4.1 , we have

$$
\left\|Q_{m p}\left(R_{\lambda^{\prime}}\right)-\nu_{r}\left(R_{\lambda} ; \tilde{a}_{1}, \ldots, \tilde{a}_{r}\right)\right\| \leq \alpha^{\prime} \widetilde{K}_{r \delta} h(\lambda)^{-3 / 2} .
$$

This implies the following result.

Corollary 4.2. Under the above conditions on the family $\left\{R_{\lambda}, \lambda>0\right\}$, if $X$ has characteristic function $\phi_{X}=\psi(\theta) r_{\lambda}$ and if (2.5) is satisfied with $r \geq 2$ and $\delta>0$, then, for $\lambda^{\prime}$, $m$ and $p$ solving (4.24) and for $t^{\prime}:=\min \{3, r+\delta\}$, we have translated $R_{\lambda}$-approximation of the form

$$
\begin{array}{ll}
\text { 1. } & d_{\mathrm{loc}}\left(P_{X}, Q_{m p}\left(R_{\lambda^{\prime}}\right)\right) \leq \gamma_{1}^{\prime} h(\lambda)^{-\left(t^{\prime}+1\right) / 2} ; \\
\text { 2. } & d_{\mathrm{K}}\left(P_{X}, Q_{m p}\left(R_{\lambda^{\prime}}\right)\right) \leq \gamma_{2}^{\prime} h(\lambda)^{-t^{\prime} / 2},
\end{array}
$$

for suitable choice of $\gamma_{1}^{\prime}, \gamma_{2}^{\prime}$. Furthermore, if (4.20) holds, then

$$
\text { 3. }\left\|P_{X}-Q_{m p}\left(R_{\lambda^{\prime}}\right)\right\| \leq \gamma_{3}^{\prime} h(\lambda)^{-t^{\prime} / 2},
$$

for suitable choice of $\gamma_{3}^{\prime}$. For given family $\left\{R_{\lambda}, \lambda>0\right\}$, the constants $\gamma_{l}^{\prime}, l=1,2,3$, are bounded for $\left(\tilde{a}_{1}, \ldots, \tilde{a}_{r}\right)$ in compact sets. 
Suppose that, instead of (2.5), we are given an approximation to $\psi$ by a Taylor expansion $\psi_{r}$ around $\theta=0$ :

$$
\left|\psi(\theta)-\psi_{r}(\theta)\right| \leq K_{r \delta}|\theta|^{r+\delta}, \quad|\theta| \leq \pi
$$

for some $0<\delta \leq 1$, where

$$
\psi_{r}(\theta):=\sum_{l=0}^{r} a_{l}(i \theta)^{l}
$$

for real coefficients $a_{l}$ (and with $a_{0}=1$ ) and some $r \in \mathbb{N}_{0}$. Then, equating coefficients of $i \theta$, it follows that

$$
\left|\psi_{r}(\theta)-\tilde{\psi}_{r}(\theta)\right| \leq G_{r}|\theta|^{r+1}, \quad|\theta| \leq \pi,
$$

for $G_{r}:=G_{r}\left(a_{1}, \ldots, a_{r}\right)$, if $\tilde{a}_{1}, \ldots, \tilde{a}_{r}$ are defined implicitly by

$$
a_{j}:=\sum_{l=1}^{j} \tilde{a}_{l} \sum_{\left(s_{1}, \ldots, s_{l}\right) \in S_{j-l}} \prod_{t=1}^{l} \frac{1}{\left(s_{t}+1\right) !}
$$

where $S_{m}:=\left\{\left(s_{1}, \ldots, s_{l}\right): \sum_{t=1}^{l} s_{t}=m\right\}$. Hence we can replace (2.5) by (4.29) in the assumptions of the theorems, if, in the conclusions, $K_{r \delta}$ is replaced by $K_{r \delta}+\pi^{1-\delta} G_{r}$.

\section{Poisson-Charlier expansions}

The most common family of distributions in applications is the Poisson family, with $R_{\lambda}=\operatorname{Po}(\lambda)$ and $r_{\lambda}=p_{\lambda}$. Here, as observed in Section 2, the bound (2.3) is satisfied with $\rho=2 \pi^{-2} \lambda$, so that Theorem 2.2 can be applied to approximate the distribution $P_{X}$ of a random variable $X$ whose characteristic function $\phi_{X}$ has the form $\psi p_{\lambda}$, if (2.5) holds for some $r$ and $\tilde{a}_{1}, \ldots, \tilde{a}_{r}$. The signed measures $\nu_{r}$ on $\mathbb{N}_{0}$ have the explicit representation

$$
\nu_{r}\{j\}:=\nu_{r}\left(\operatorname{Po}(\lambda) ; \tilde{a}_{1}, \ldots, \tilde{a}_{r}\right)\{j\}:=\operatorname{Po}(\lambda)\{j\}\left\{1+\sum_{l=1}^{r}(-1)^{l} \tilde{a}_{l} C_{l}(j ; \lambda)\right\},
$$

where

$$
C_{l}(j ; \lambda):=\sum_{k=0}^{l}(-1)^{k}\left(\begin{array}{l}
l \\
k
\end{array}\right)\left(\begin{array}{l}
j \\
k
\end{array}\right) k ! \lambda^{-k}
$$

denotes the l-th Charlier polynomial (Chihara 1978, (1.9), p. 171).

Note that, if $\left(\begin{array}{l}j \\ k\end{array}\right)$ is replaced by $j^{k} / k$ ! in (5.2), one obtains the binomial expansion of $(1-j / \lambda)^{l}$. As this suggests, the values of $C_{l}(j ; \lambda)$ are in fact small for $j$ near $\lambda$ if $\lambda$ is large:

$$
\left|C_{l}(j ; \lambda)\right| \leq 2^{l-1}\left\{|1-j / \lambda|^{l}+(l / \sqrt{\lambda})^{l}\right\}
$$

(Barbour \& Čekanavičius 2002, Lemma 6.1). (5.3) thus implies that, in any interval of the form $|j-\lambda| \leq c \sqrt{\lambda}$, which is where the probability mass of $\operatorname{Po}(\lambda)$ is mostly to be found, the correction to the Poisson measure $\operatorname{Po}(\lambda)$ is of uniform relative order $O\left(\lambda^{-l / 2}\right)$. Indeed, the Chernoff inequalities for $Z \sim \operatorname{Po}(\lambda)$ can be expressed in the form

$$
\begin{aligned}
\max & \{\mathbb{P}[Z>\lambda(1+\delta)], \mathbb{P}[Z<\lambda(1-\delta)]\} \\
& \leq \exp \left\{-\lambda \delta^{2} / 2(1+\delta / 3)\right\} \leq \exp \left\{-\lambda \delta^{2} / 3(\delta \vee 1)\right\},
\end{aligned}
$$

(Chung \& Lu 2006, Theorem 3.2). Since also, from (5.2),

$$
\left|C_{l}(j ; \lambda)\right| \leq(1+j / \lambda)^{l} \leq 2^{l} \quad \text { if } \quad 0 \leq j \leq \lambda
$$


and since

$$
\left(\begin{array}{l}
j \\
k
\end{array}\right) k ! \lambda^{-k} \frac{e^{-\lambda} \lambda^{j}}{j !}=\frac{e^{-\lambda} \lambda^{j-k}}{(j-k) !} \leq \frac{e^{-\lambda} \lambda^{j-l}}{(j-l) !}
$$

if $0 \leq k \leq l$ and $j \geq l+\lambda$, it follows that, for any $l \geq 0$, we have

$$
\sum_{j=0}^{m}\left|C_{l}(j ; \lambda)\right| \operatorname{Po}(\lambda)\{j\} \leq 2^{l} \mathbb{P}[Z \leq m] \leq 2^{l} \exp \left\{-(\lambda-m)^{2} / 3 \lambda\right\}
$$

for $m \leq \lambda$, and, for $l \leq r$ and $m \geq \lambda+r$,

$$
\begin{aligned}
\sum_{j \geq m}\left|C_{l}(j ; \lambda)\right| \operatorname{Po}(\lambda)\{j\} \leq 2^{l} \mathbb{P}[Z \geq m-l] \leq 2^{l} \mathbb{P}[Z \geq m-r] \\
\leq 2^{l}\left\{\exp \left\{-(m-r-\lambda)^{2} / 3 \lambda\right\} \vee \exp \{-(m-r-\lambda) / 3\}\right\} .
\end{aligned}
$$

It thus follows that

$$
\begin{aligned}
\left|\nu_{r}\right|\{[0, m]\} & \leq \bar{A}_{r} e^{-(\lambda-m)^{2} / 3 \lambda}, \quad 0 \leq m \leq \lambda ; \\
\left|\nu_{r}\right|\{[m, \infty)\} & \leq \bar{A}_{r}\left\{e^{-(m-r-\lambda)^{2} / 3 \lambda} \vee e^{-(m-r-\lambda) / 3}\right\}, \quad m \geq \lambda+r,
\end{aligned}
$$

where $\bar{A}_{r}:=1+\sum_{l=1}^{r} 2^{l}\left|\tilde{a}_{l}\right|$, demonstrating concentration of measure for $\nu_{r}$ on a scale of $\sqrt{\lambda}$ around $\lambda$. Moreover, it can be deduced from (5.3) that there exists a positive constant $d=d\left(\tilde{a}_{1}, \ldots, \tilde{a}_{r}\right)$ such that $\nu_{r}\{j\} \geq 0$ for $|j-\lambda| \leq d \lambda$, and it follows from (5.5) that $\left|\nu_{r}\right|\{j:|j-\lambda|>d \lambda\}=O\left(e^{-\alpha \lambda}\right)$ for some $\alpha>0$. Since also $\nu_{r}\left\{\mathbb{N}_{0}\right\}=1$, it thus follows that, even if $\nu_{r}$ is formally a signed measure, it differs from a probability only on a set of measure exponentially small with $\lambda$.

If (2.5) holds, Theorem 2.2 enables one to deduce simple bounds for $d_{\mathrm{loc}}\left(P_{X}, \nu_{r}\right)$ and $d_{\mathrm{K}}\left(P_{X}, \nu_{r}\right)$. For the total variation norm, with judicious choice of $m_{1}$ and $m_{2}$, we can use part 1 of Theorem 2.2 to bound

$$
\sum_{j=m_{1}+1}^{m_{2}-1}\left|P_{X}\{j\}-\nu_{r}\{j\}\right| \leq\left(m_{2}-m_{1}-1\right) \sup _{j \in \mathbb{N}_{0}}\left|P_{X}\{j\}-\nu_{r}\{j\}\right|,
$$

and then (5.5) and part 2 to take care of the remaining tail probabilities:

$$
\begin{aligned}
\sum_{j \leq m_{1}} \mid P_{X}\{j\}-\nu_{r} & \{j\}\left|\leq P_{X}\left\{\left(-\infty, m_{1}\right]\right\}+\right| \nu_{r} \mid\left\{\left[0, m_{1}\right]\right\} \\
& \leq \sup _{l \in \mathbb{N}_{0}}\left|P_{X}\{(-\infty, l]\}-\nu_{r}\{[0, l]\}\right|+2\left|\nu_{r}\right|\left\{\left[0, m_{1}\right]\right\},
\end{aligned}
$$

and

$$
\begin{aligned}
& \sum_{j \geq m_{2}}\left|P_{X}\{j\}-\nu_{r}\{j\}\right| \leq P_{X}\left\{\left[m_{2}, \infty\right)\right\}+\left|\nu_{r}\right|\left\{\left[m_{2}, \infty\right)\right\} \\
& \quad \leq \sup _{l \in \mathbb{N}_{0}}\left|P_{X}\{(-\infty, l]\}-\nu_{r}\{[0, l]\}\right|+2\left|\nu_{r}\right|\left\{\left[m_{2}, \infty\right)\right\} .
\end{aligned}
$$

This gives the following theorem.

Theorem 5.1. Suppose that $X$ is as above, having characteristic function $\phi_{X}:=\psi p_{\lambda}$, and that (2.5) holds; write $t=r+\delta$ as before. If $\lambda \geq 1$, there is a constant $\alpha_{4 t}=\alpha_{4 t}\left(\tilde{a}_{0}, \ldots, \tilde{a}_{r}\right)$ such that

$$
\left\|P_{X}-\nu_{r}\right\| \leq \alpha_{4 t} K_{r \delta}(\lambda \vee 1)^{-t / 2} \max \left\{1, \sqrt{\left|\log K_{r \delta}\right|}, \sqrt{\log (\lambda+1)}\right\} ;
$$


if $\lambda<1$, then there is a constant $\alpha_{5 t}=\alpha_{5 t}\left(\tilde{a}_{0}, \ldots, \tilde{a}_{r}\right)$ such that

$$
\left\|P_{X}-\nu_{r}\right\| \leq \alpha_{5 t} K_{r \delta} \lambda^{-t / 2} \max \left\{1,\left|\log K_{r \delta}\right|\right\} .
$$

Proof. For $\lambda \geq 1$, we use both parts of (5.5), with

$$
m_{1}:=\left\lfloor\lambda-c_{r \lambda} \sqrt{\lambda \log (\lambda+1)}\right\rfloor \text { and } m_{2}:=\left\lceil\lambda+r+c_{r \lambda} \sqrt{\lambda \log (\lambda+1)}\right\rceil,
$$

where $\lfloor x\rfloor \leq x \leq\lceil x\rceil$ denote the integers closest to $x$, and with

$$
c_{r \lambda}:=3\left\{(r+1) / 2+\left|\log K_{r \delta}\right| / \log (\lambda+1)\right\} .
$$

If $r+c_{r \lambda} \sqrt{\lambda \log (\lambda+1)} \leq \lambda$, we obtain

$$
\left|\nu_{r}\right|\left\{\left[m_{1}, m_{2}\right]^{c}\right\} \leq 2 \bar{A}_{r}(\lambda+1)^{-c_{r \lambda}^{2} / 3} \leq 2 \bar{A}_{r}(\lambda+1)^{-c_{r \lambda} / 3},
$$

since $c_{r \lambda} \geq 1$, and, if $r+c_{r \lambda} \sqrt{\lambda \log (\lambda+1)}>\lambda$, we get

$$
\left|\nu_{r}\right|\left\{\left[m_{1}, m_{2}\right]^{c}\right\} \leq 2 \bar{A}_{r} \exp \left\{-c_{r \lambda} \sqrt{\lambda \log (\lambda+1)} / 3\right\} \leq 2 \bar{A}_{r}(\lambda+1)^{-c_{r \lambda} / 3},
$$

since $\lambda \geq \log (\lambda+1)$ in $\lambda \geq 0$. Hence, in either case, from the definition of $c_{r \lambda}$, we have

$$
\left|\nu_{r}\right|\left\{\left[m_{1}, m_{2}\right]^{c}\right\} \leq 2 \bar{A}_{r} K_{r \delta}(\lambda+1)^{-(r+1) / 2} .
$$

Hence, from Theorem 2.2 and (5.6)-(5.8), it follows that

$$
\begin{gathered}
\left\|P_{X}-\nu_{r}\right\| \leq\left\{2 c_{r \lambda} \sqrt{\lambda \log (\lambda+1)}+r+2\right\} \alpha_{1 t}^{\prime} K_{r \delta} \lambda^{-(t+1) / 2} \\
+2 \alpha_{2 t}^{\prime} K_{r \delta} \lambda^{-t / 2}+4 \bar{A}_{r} K_{r \delta} \lambda^{-(r+1) / 2}
\end{gathered}
$$

with

$$
\alpha_{1 t}^{\prime}:=\alpha_{1 t}\left(\pi^{2} / 2\right)^{(t+1) / 2} ; \quad \alpha_{2 t}^{\prime}:=\alpha_{2 t}\left(\pi^{2} / 2\right)^{t / 2}
$$

so that

$$
\left\|P_{X}-\nu_{r}\right\| \leq \beta_{3 t} K_{r \delta} \lambda^{-t / 2} \sqrt{\log (\lambda+1)} \max \left\{1, \frac{\left|\log K_{r \delta}\right|}{\log (\lambda+1)}\right\},
$$

with $\beta_{3 t}:=\alpha_{1 t}^{\prime}\{4 r+11\}+2 \alpha_{2 t}^{\prime}+4 \bar{A}_{r}$.

For $\lambda<1$, we take $m_{2}:=\left\lceil 2+r+3\left|\log K_{r \delta}\right|\right\rceil$ in (5.5), giving

$$
\left|\nu_{r}\right|\left\{\left[m_{2}, \infty\right)\right\} \leq \bar{A}_{r} K_{r \delta},
$$

and then, from Theorem 2.2, (5.6) and (5.8), it follows that

$$
\left\|P_{X}-\nu_{r}\right\| \leq\left(r+3+3\left|\log K_{r \delta}\right|\right) \alpha_{1 t}^{\prime} K_{r \delta}+2 \alpha_{2 t}^{\prime} K_{r \delta}+2 \bar{A}_{r} K_{r \delta}
$$

so that

$$
\left\|P_{X}-\nu_{r}\right\| \leq \beta_{3 t}^{\prime} K_{r \delta} \max \left\{1, \mid \log \left(K_{r \delta} \mid\right\},\right.
$$

with $\beta_{3 t}^{\prime}:=\alpha_{1 t}^{\prime}\{r+6\}+2 \alpha_{2 t}^{\prime}+2 \bar{A}_{r}$.

Sometimes it is convenient, for simplicity, to use parameters in the expansions that are not those emerging naturally from the proofs. The following theorem shows that such alterations can easily be allowed for. 
Theorem 5.2. Suppose that

$$
\phi_{\mu}:=p_{\lambda} A ; \quad \phi_{\nu^{(1)}}:=p_{\lambda} A^{\prime} ; \quad \phi_{\nu^{(2)}}:=p_{\lambda^{\prime}} A,
$$

with $A(\theta):=1+\sum_{l=1}^{r} a_{l} \theta^{l}, A^{\prime}(\theta):=1+\sum_{l=1}^{r} a_{l}^{\prime} \theta^{l}$ and with $\lambda>\lambda^{\prime}$. Then, with $\rho:=2 \pi^{-2} \lambda$, $\rho^{\prime}:=2 \pi^{-2} \lambda^{\prime}$ and $a_{0}:=1$,

$$
\begin{aligned}
d_{\mathrm{loc}}\left(\mu, \nu^{(1)}\right) & \leq \sum_{l=1}^{r} \alpha_{1 l}\left|a_{l}-a_{l}^{\prime}\right|(\rho \vee 1)^{-(l+1) / 2} ; \\
d_{\mathrm{K}}\left(\mu, \nu^{(1)}\right) & \leq \sum_{l=1}^{r} \alpha_{2 l}\left|a_{l}-a_{l}^{\prime}\right|(\rho \vee 1)^{-l / 2} ; \\
d_{\mathrm{loc}}\left(\mu, \nu^{(2)}\right) & \leq\left(\lambda-\lambda^{\prime}\right) \sum_{l=1}^{r+1} \alpha_{1 l}\left|a_{l-1}\right|\left(\rho^{\prime} \vee 1\right)^{-(l+1) / 2} ; \\
d_{\mathrm{K}}\left(\mu, \nu^{(2)}\right) & \leq\left(\lambda-\lambda^{\prime}\right) \sum_{l=1}^{r+1} \alpha_{2 l}\left|a_{l-1}\right|\left(\rho^{\prime} \vee 1\right)^{-l / 2} .
\end{aligned}
$$

Proof. For the comparison between $\mu$ and $\nu^{(1)}$, we have

$$
\left|A(\theta)-A^{\prime}(\theta)\right| \leq \sum_{l=1}^{r}\left|a_{l}-a_{l}^{\prime}\right||\theta|^{l}, \quad 0<|\theta| \leq \pi,
$$

and Proposition 2.1 completes the proof. For that between $\mu$ and $\nu^{(2)}$, note that $p_{\lambda}=$ $p_{\lambda-\lambda^{\prime}} p_{\lambda^{\prime}}$, and that, for $\lambda>\lambda^{\prime}$ and $0<|\theta| \leq \pi$,

$$
\left|p_{\lambda-\lambda^{\prime}}(\theta)-1\right||A(\theta)| \leq\left(\lambda-\lambda^{\prime}\right)|\theta|\left\{1+\sum_{l=1}^{r}\left|a_{l}\right||\theta|^{l}\right\},
$$

from which and Proposition 2.1 the remaining results follow.

\section{Poisson approximation}

The measures $\nu_{r}$ used in the approximations of the previous section are very explicit. However, approximation in terms of a Poisson distribution is more convenient, if possible. Clearly, if (2.5) holds for any $r=r^{*}, \delta=\delta^{*}$, then it holds with $r=0$ and $\psi_{0}(\theta)=1$ for all $\theta$, with the exponent $r+\delta$ replaced by $\delta_{0}=\delta^{*}$ if $r^{*}=0$ and by $\delta_{0}=1$ if $r^{*} \geq 1$, with $K_{0 \delta_{0}}$ depending on $r^{*}, K_{r^{*} \delta^{*}}$ and $\tilde{a}_{1}, \ldots, \tilde{a}_{r^{*}}$. Theorem 2.2 then gives approximation by $\operatorname{Po}(\lambda)$ with accuracy in Kolmogorov distance of order $O\left(\lambda^{-t_{0} / 2}\right)$, for $t_{0}=\min \left\{1, r^{*}+\delta^{*}\right\}$.

However, if $r^{*} \geq 1$, one can also write

$$
\psi(\theta) p_{\lambda}(\theta)=\hat{\psi}(\theta) p_{\lambda^{\prime}}(\theta)
$$

for any $\lambda^{\prime}>0$, where

$$
\hat{\psi}(\theta):=\psi(\theta) \exp \left\{\left(\lambda-\lambda^{\prime}\right)\left(e^{i \theta}-1\right)\right\} .
$$

Taking $\lambda^{\prime}-\lambda=\tilde{a}_{1}$ now gives a bound

$$
|\hat{\psi}(\theta)-1| \leq K_{1 \delta_{1}}|\theta|^{t_{1}}, \quad 0 \leq|\theta| \leq \pi,
$$

of the form (2.5), with $\delta_{1}=\min \left\{r^{*}+\delta^{*}-1,1\right\}, t_{1}=1+\delta_{1}$ and with $K_{1 \delta_{1}}$ depending on $r^{*}, K_{r^{*}}$ and $\tilde{a}_{1}, \ldots, \tilde{a}_{r^{*}}$. Hence, Theorem 2.2 implies the following approximation. 
Corollary 6.1. If $X$ has characteristic function $\phi_{X}=\psi p_{\lambda}$ such that (2.5) is satisfied with $r \geq 1$ and $0<\delta \leq 1$, then we have

$$
\begin{array}{ll}
\text { 1. } & d_{\mathrm{loc}}\left(P_{X}, \operatorname{Po}\left(\lambda^{\prime}\right)\right) \leq \alpha_{1 t^{\prime}} K_{1, t^{\prime}-1}\left(\rho^{\prime} \vee 1\right)^{-\left(t^{\prime}+1\right) / 2} ; \\
\text { 2. } & d_{\mathrm{K}}\left(P_{X}, \operatorname{Po}\left(\lambda^{\prime}\right)\right) \leq \alpha_{2 t^{\prime}} K_{1, t^{\prime}-1}\left(\rho^{\prime} \vee 1\right)^{-t^{\prime} / 2},
\end{array}
$$

where $\lambda^{\prime}=\lambda+\tilde{a}_{1}, t^{\prime}:=\min \{2, r+\delta\}$ and $\rho^{\prime}=2 \pi^{-2} \lambda^{\prime}$, for a suitable constant $K_{1, t^{\prime}-1}$ depending on $r, K_{r \delta}$ and $\tilde{a}_{1}, \ldots, \tilde{a}_{r}$.

The parameter $\lambda^{\prime}$ is chosen to make the Poisson mean $\lambda^{\prime}$ equal to the mean $\lambda+a_{1}$ of $X$. This choice of the Poisson parameter improves the rate, in the asymptotic sense that, if $a_{1}, \ldots, a_{r}$ and $K_{r \delta}$ remain bounded but $\lambda \rightarrow \infty$, and if $r+\delta \geq 2$, then the approximation error for Kolmogorov distance is of order $O\left(\lambda^{-1}\right)$, as opposed to the rate of order $O\left(\lambda^{-1 / 2}\right)$ in general obtained when approximating by $\operatorname{Po}(\lambda)$.

Analogously, fitting the second moment as well (if it is finite) can lead to asymptotically sharper approximation within the translated Poisson family. The corresponding result has already been proved in Corollary 4.2 under the more general assumptions of Section 3, which the Poisson family satisfies. If $X$ has characteristic function $\phi_{X}=\psi p_{\lambda}$ such that (2.5) is satisfied with $r \geq 2$ and $0<\delta \leq 1$, then the first two moments of $Q_{m p}\left(\operatorname{Po}\left(\lambda^{\prime}\right)\right)$ match those of $X$ if

$$
\lambda^{\prime}+m+p=\lambda+\tilde{a}_{1} ; \quad \lambda^{\prime}+p(1-p)=\lambda+2 \tilde{a}_{2}+\tilde{a}_{1}\left(1-\tilde{a}_{1}\right) .
$$

$\lambda^{\prime}, m$ and $p$ are found as prescribed following (4.24), giving

$$
\begin{aligned}
m & =\left\lfloor\tilde{a}_{1}^{2}-2 \tilde{a}_{2}\right\rfloor ; \quad p^{2}=\left\langle\tilde{a}_{1}^{2}-2 \tilde{a}_{2}\right\rangle ; \\
\lambda^{\prime} & =\lambda+2 \tilde{a}_{2}+\tilde{a}_{1}\left(1-\tilde{a}_{1}\right)-p(1-p),
\end{aligned}
$$

where $\langle x\rangle$ denotes the fractional part of $x$. Corollary 4.2 now yields the following result.

Corollary 6.2. If $X$ has characteristic function $\phi_{X}=\psi p_{\lambda}$ such that (2.5) is satisfied with $r \geq 2$ and $0<\delta \leq 1$, then for $\lambda^{\prime}, m$ amd $p$ given in (6.1) and for $t^{\prime}:=\min \{3, r+\delta\}$, we have translated Poisson approximation:

$$
\begin{array}{ll}
\text { 1. } & d_{\mathrm{loc}}\left(P_{X}, Q_{m p}\left(\operatorname{Po}\left(\lambda^{\prime}\right)\right)\right) \leq \gamma_{1}^{\prime}(\lambda \vee 1)^{-\left(t^{\prime}+1\right) / 2} ; \\
\text { 2. } & d_{\mathrm{K}}\left(P_{X}, Q_{m p}\left(\operatorname{Po}\left(\lambda^{\prime}\right)\right)\right) \leq \gamma_{2}^{\prime}(\lambda \vee 1)^{-t^{\prime} / 2},
\end{array}
$$

and, if (4.20) holds, then

$$
\text { 3. }\left\|P_{X}-Q_{m p}\left(\operatorname{Po}\left(\lambda^{\prime}\right)\right)\right\| \leq \gamma_{3}^{\prime}(\lambda \vee 1)^{-t^{\prime} / 2}
$$

for constants $\gamma_{l}^{\prime}=\gamma_{l}^{\prime}\left(\tilde{a}_{1}, \ldots, \tilde{a}_{r}\right), l=1,2,3$, that are bounded for $\left(\tilde{a}_{1}, \ldots, \tilde{a}_{r}\right)$ in compact sets.

In particular, if $a_{1}, \ldots, a_{r}$ and $K_{r \delta}$ remain bounded but $\lambda \rightarrow \infty$, and if $r+\delta \geq 3$, then $t^{\prime}=3$ and the order of approximation in Kolmogorov distance is of order $O\left(\lambda^{-3 / 2}\right)$.

\section{Compound Poisson approximation}

The theory of Section 3 can also be applied when the distributions $R_{\lambda}$ come from a compound Poisson family. For $\lambda>0$ and for $\mu$ a probability distribution on $\mathbb{Z}$, let $\operatorname{CP}(\lambda, \mu)$ denote the distribution of the sum $Y:=\sum_{j \in \mathbb{Z} \backslash\{0\}} j Z_{j}$, where $Z_{j}, j \neq 0$, are independent, and $Z_{j} \sim \operatorname{Po}\left(\lambda \mu_{j}\right)$. Then, if $\mu_{1}>0$, the characteristic function of $Y$ is of the form $R_{\lambda}:=\zeta_{\lambda} p_{\lambda_{1}}$, where $\zeta_{\lambda}$ is the characteristic function of $\sum_{j \in \mathbb{Z} \backslash\{0,1\}} j Z_{j}$ and $\lambda_{1}=\lambda \mu_{1}$. Thus, for the purposes of applying Corollaries 4.1 and $4.2, \rho$ can be taken 
to be $2 \pi^{-2} \mu_{1} \lambda$. Corollary 4.2 , for instance, then gives conditions under which translated compound Poisson distribution can be achieved, with approximation at rate $O\left(\lambda^{-3 / 2}\right)$.

These considerations apply as long as $\mu_{1}>0$, and could also be invoked if $\mu_{-1}>$ 0 . If $\mu_{1}=\mu_{-1}=0$, there is then no factor of the form $p_{\lambda}$ to guarantee that, for some $\rho>0$, the characteristic function $\phi_{Y}$ of $Y$ (corresponding to the characteristic function $\chi$ of Proposition 2.1) satisfies $\left|\phi_{Y}(\theta)\right| \leq \exp \left\{-\rho \theta^{2}\right\}$ for all $|\theta| \leq \pi$. Some additional aperiodicity condition needs to be satisfied, if the family $\{\mathrm{CP}(\lambda, \mu), \lambda \geq 1\}$ is to satisfy (4.19). Indeed, if $Y=2 Z$ where $Z \sim \operatorname{Po}(\lambda)$, and if $W \sim \operatorname{Be}(1 / 2)$ is independent of $Y$, it is not true that the distribution of $Y+W$ is close to that of $Y$ in total variation, even though $\left|\phi_{Y+W}(\theta)-\phi_{Y}(\theta)\right| \leq K_{0}|\theta|\left|\phi_{Y}(\theta)\right|$.

\section{Applications}

8.1. Sums of independent random variables. Let $X_{1}, \ldots, X_{n}$ be independent integer valued random variables, and let $S_{n}$ denote their sum. In contexts in which a central limit approximation to the distribution of $S_{n}$ would be appropriate, the classical Edgeworth expansion (see, e.g., Petrov 1975, Chapter 5) is unwieldy, because $S_{n}$ is confined to the integers. As an alternative, Barbour and Cekanavičius (2002, Theorem 5.1) give a Poisson-Charlier expansion, for $S_{n}$ 'centred' so that its mean and variance are almost equal, with an error bound expressed in the total variation norm. Here, we show that an error bound for their expansion of the same order can be established by the techniques of this paper, but with respect to the less stringent Kolmogorov distance.

Assume that each of the $X_{j}$ has finite $(r+1+\delta)^{\prime}$ th moment, with $r \geq 1$, and define

$$
A^{(r)}(w):=1+\sum_{l \geq 2} \tilde{a}_{l}^{(r)} w^{l}=\exp \left\{\sum_{l=2}^{r+1} \frac{\kappa_{l} w^{l}}{l !}\right\},
$$

where $\kappa_{l}:=\kappa_{l}\left(S_{n}\right)$ and $\kappa_{l}(X)$ denotes the l'th factorial cumulant of the random variable $X$. Then the approximation that we establish is to the Poisson-Charlier signed measure $\nu_{r}$ with

$$
\nu_{r}\{j\}:=\operatorname{Po}(\lambda)\{j\}\left\{1+\sum_{l=2}^{L_{r}}(-1)^{l} \tilde{a}_{l}^{(r)} C_{l}(j ; \lambda)\right\},
$$

where $L_{r}:=\max \{1,3(r-1)\}$, and where $\lambda:=\mathbb{E} S_{n} ; \nu_{r}$ has characteristic function

$$
\phi_{\nu_{r}}:=p_{\lambda}(\theta) \widetilde{A}^{(r)}(\theta)
$$

where

$$
\widetilde{A}^{(r)}(\theta):=1+\sum_{l=2}^{L_{r}} \tilde{a}_{l}^{(r)}\left(e^{i \theta}-1\right)^{l} .
$$

We need two further quantities involving the $X_{j}$ :

$$
K^{(n)}:=\left|\sum_{j=1}^{n} \kappa_{2}\left(X_{j}\right)\right|=\left|\operatorname{Var} S_{n}-\mathbb{E} S_{n}\right|,
$$

and

$$
p_{j}:=1-\frac{1}{2}\left\|\mathcal{L}\left(X_{j}\right)-\mathcal{L}\left(X_{j}+1\right)\right\|
$$


Theorem 8.1. Suppose that there are constants $K_{l}, 1 \leq l \leq r+1$, such that, for each $j$,

$$
\left|\kappa_{l}\left(X_{j}\right)\right| \leq K_{l}, \quad 2 \leq l \leq r+1 ; \quad \mathbb{E}\left|X_{j}\right|^{r+1+\delta} \leq K_{1}^{r+1+\delta} .
$$

Suppose also that $p_{j} \geq p_{0}>0$ for all $j$, and that $\lambda \geq n \lambda_{0}$. Then

$$
d_{\mathrm{K}}\left(\mathcal{L}\left(S_{n}\right), \nu_{r}\right) \leq G\left(K_{1}, \ldots, K_{r+1}, K^{(n)}, p_{0}^{-1}, \lambda_{0}^{-1}\right) n^{-(r-1+\delta) / 2},
$$

for a function $G$ that is bounded on compact sets.

Remark. For asymptotics in $n$, with triangular arrays of variables, the error is of order $O\left(n^{-(r-1+\delta) / 2}\right)$ when $\lambda_{0}$ and $p_{0}$ are bounded away from zero, and $K_{1}, \ldots, K_{r+1}$ and $K^{(n)}$ remain bounded. The requirements on $\lambda_{0}$ and $p_{0}$ can often be achieved by grouping the random variables appropriately, though attention then has to be paid to the consequent changes in the $K_{l}$. The final condition can always be satisfied with $K^{(n)} \leq 1$, by replacing the $X_{j}$ by translates, where necessary. For more discussion, we refer to Barbour and Cekanavičius (2002). The above conditions are designed to cover sums of independent random variables, each of which has non-trivial variance, has uniformly bounded $(r+1+\delta)^{\prime}$ 'th moment, and whose distribution overlaps with its unit translate.

Proof. We check the conditions of Proposition 3.1. First, in view of (8.6), we can write

$$
\mathbb{E}\left(e^{i \theta X_{j}}\right)=\frac{1}{2} p_{j}\left(e^{i \theta}+1\right) \phi_{1 j}(\theta)+\left(1-p_{j}\right) \phi_{2 j}(\theta),
$$

where both $\phi_{1 j}$ and $\phi_{2 j}$ are characteristic functions. Hence we have

$$
\left|\mathbb{E}\left(e^{i \theta X_{j}}\right)\right| \leq 1-p_{j}+p_{j} \cos (\theta / 2) \leq 1-p_{j} \theta^{2} / 4 \pi, \quad 0 \leq|\theta| \leq \pi
$$

Hence $\phi_{\mu}(\theta):=\mathbb{E}\left(e^{i \theta S_{n}}\right)$ satisfies

$$
\left|\phi_{\mu}(\theta)\right| \leq \exp \left\{-n p_{0} \theta^{2} / 4 \pi\right\}, \quad 0 \leq|\theta| \leq \pi .
$$

On the other hand, from the additivity of the factorial cumulants, we have

$$
\left|\kappa_{l}\left(S_{n}\right)\right| \leq n K_{l}, \quad 3 \leq l \leq r+1,
$$

with $\left|\kappa_{2}\left(S_{n}\right)\right| \leq K^{(n)}$ from (8.5). From (8.1), we thus deduce the bound $\left|\tilde{a}_{l}^{(r)}\right| \leq c_{l} n^{\lfloor l / 3\rfloor}$, for $c_{l}=c_{l}\left(K^{(n)}, K_{3}, \ldots, K_{r+1}\right), l \geq 1$. Hence

$$
\left|\phi_{\nu_{r}}(\theta)\right| \leq \exp \left\{-2 n \lambda_{0} \theta^{2} / \pi^{2}\right\} c^{\prime} n^{\left\lfloor L_{r} / 3\right\rfloor} \leq \exp \left\{-n \lambda_{0} \theta^{2} / \pi^{2}\right\} c^{\prime \prime},
$$

for $c^{\prime \prime}=c^{\prime \prime}\left(K^{(n)}, K_{3}, \ldots, K_{r+1}\right)$. Combining (8.7) and (8.8), we can thus take $\eta:=$ $C e^{-n \rho^{\prime} \theta_{0}^{2}}$ in Proposition 3.1, for

$$
\rho^{\prime}=\min \left\{\lambda_{0} / \pi^{2}, p_{0} / 4 \pi\right\}
$$

and a suitable $C=C\left(K^{(n)}, K_{3}, \ldots, K_{r+1}\right)$. The choice of $\theta_{0}$ we postpone for now.

For $|\theta| \leq \theta_{0}$, we take $\chi(\theta):=p_{\lambda}(\theta)$, and check the approximation of

$$
\phi_{\mu}(\theta) \exp \left\{-\lambda\left(e^{i \theta}-1\right)\right\}=\mathbb{E}\left\{(1+w)^{S_{n}}\right\} e^{-w \mathbb{E} S_{n}}
$$

by $\widetilde{A}^{(r)}(\theta)$ as a polynomial in $w:=e^{i \theta}-1$. We begin with the inequality

$$
\begin{aligned}
\left|(1+w)^{s}-\sum_{l=0}^{r+1} \frac{w^{l}}{l !} s_{(l)}\right| & \leq \frac{\left|s_{(r+2)}\right|}{(r+2) !}|w|^{r+2} \wedge 2 \frac{\left|s_{(r+1)}\right|}{(r+1) !}|w|^{r+1} \\
& \leq \frac{\left|s_{(r+1)}\right|}{(r+2) !}|w|^{r+1+\delta}\{|s|+r+1\}^{\delta}\{2(r+2)\}^{1-\delta},
\end{aligned}
$$


derived using Taylor's expansion, true for any $s \in \mathbb{Z}$ and $0<\delta \leq 1$, where $s_{(l)}:=$ $s(s-1) \ldots(s-l+1)$. Hence, for each $j$, we have

$$
\left|\mathbb{E}\left\{(1+w)^{X_{j}}\right\}-\sum_{l=0}^{r+1} \frac{\mathbb{E}\left\{\left(X_{j}\right)_{(l)}\right\}}{l !} w^{l}\right| \leq c_{r, \delta}|\theta|^{r+1+\delta}\left(K_{1}+K_{1}^{r+1+\delta}\right),
$$

for a universal constant $c_{r, \delta}$. Then, writing

$$
Q_{r+1}^{(s)}(w ; X):=\exp \left\{\sum_{l=s}^{r+1} \kappa_{l}(X) w^{l} / l !\right\}
$$

and using the differentiation formula in Petrov (1975, p. 170), we have

$$
\begin{aligned}
\mid Q_{r+1}^{(1)}\left(w ; X_{j}\right) & -\sum_{l=0}^{r+1} \frac{\mathbb{E}\left\{\left(X_{j}\right)_{(l)}\right\}}{l !} w^{l} \mid \\
& \leq \frac{|\theta|^{r+2}}{(r+2) !} \sup _{\left|\theta^{\prime}\right| \leq \theta_{0}}\left|\frac{d^{r+2}}{d z^{r+2}} Q_{r+1}^{(1)}\left(z ; X_{j}\right)\right|_{z=e^{i \theta^{\prime}-1}} \\
& \leq|\theta|^{r+2} c\left(K_{1}, \ldots, K_{r+1}\right),
\end{aligned}
$$

for a suitable function $c$ and for all $|\theta| \leq \pi$. Combining these estimates, we deduce that, for $w=e^{i \theta}-1$ and for all $|\theta| \leq \pi$,

$$
\left|\mathbb{E}\left\{(1+w)^{X_{j}}\right\} e^{-\mathbb{E} X_{j} w}-Q_{r+1}^{(2)}\left(w ; X_{j}\right)\right| \leq k_{1}|\theta|^{r+1+\delta},
$$

where $k_{1}=k_{1}\left(K_{1}, \ldots, K_{r+1}\right)$.

Now a standard inequality shows that, for $u_{j}:=\prod_{l=1}^{j} x_{l} \prod_{l=j+1}^{n} y_{l}$, for complex $x_{l}, y_{l}$ with $y_{l} \neq 0$ and $\left|x_{l} / y_{l}-1\right| \leq \varepsilon_{l}$, then

$$
\left|u_{n}-u_{0}\right| \leq\left|u_{0}\right|\left\{\prod_{s=1}^{n-1}\left(1+\varepsilon_{s}\right)\right\} \sum_{l=1}^{n} \varepsilon_{l} .
$$

Taking $x_{j}:=\mathbb{E}\left\{(1+w)^{X_{j}}\right\} e^{-\mathbb{E} X_{j} w}$ and $y_{j}:=Q_{r+1}^{(2)}\left(w ; X_{j}\right),(8.11)$ shows that we can take $\varepsilon_{l}:=\varepsilon:=k_{1}|\theta|^{r+1+\delta} e^{E}$ for each $l$, with

$$
E:=\exp \left\{\sum_{l=2}^{r+1} K_{l} / l !\right\}
$$

provided that $|\theta| \leq \theta_{0} \leq 1$. For $r \geq 2$, choosing $\theta_{0}:=n^{-1 / 3}$ then ensures that $(1+\varepsilon)^{n}$ is suitably bounded, and (8.12) yields

$$
\left|\mathbb{E}\left\{(1+w)^{S_{n}}\right\} e^{-w \mathbb{E} S_{n}}-Q_{r+1}^{(2)}\left(w ; S_{n}\right)\right| \leq k_{2} n|\theta|^{r+1+\delta},
$$

for $k_{2}=k_{2}\left(K^{(n)}, K_{1}, \ldots, K_{r+1}\right)$, since

$$
\left|u_{0}\right|:=\left|Q_{r+1}^{(2)}\left(w ; S_{n}\right)\right| \leq \exp \left\{\left|\kappa_{2}\left(S_{n}\right)\right| \theta_{0}^{2} / 2\right\} \exp \left\{\sum_{l=3}^{r+1} n K_{l} \theta_{0}^{l} / l !\right\}
$$

is bounded for $\theta_{0}=n^{-1 / 3}$, in view of (8.5). For $r=1,\left|u_{0}\right|$ is uniformly bounded if $\theta_{0} \leq 1$, and the choice $\theta_{0}=n^{-1 /(2+\delta)}$ ensures that $(1+\varepsilon)^{n}$ remains bounded. 
The remaining step is to note that, for $w:=e^{i \theta}-1, \widetilde{A}^{(r)}(\theta)$ contains all terms up to the power $w^{L_{r}}$ in the power series expansion of $Q_{r+1}^{(2)}\left(w ; S_{n}\right)$, giving

$$
\left|Q_{r+1}^{(2)}\left(w ; S_{n}\right)-\widetilde{A}^{(r)}(\theta)\right| \leq \frac{|\theta|^{L_{r}+1}}{\left(L_{r}+1\right) !} \sup _{\left|\theta^{\prime}\right| \leq|\theta|}\left|\frac{d^{L_{r}+1}}{d z^{L_{r}+1}} Q_{r+1}^{(2)}\left(z ; S_{n}\right)\right|_{z=e^{i \theta^{\prime}-1}} .
$$

Now $\left|\kappa_{2}\left(S_{n}\right)\right|$ is bounded by $K^{(n)}$, and, for $l \geq 3$, each $\kappa_{l}\left(S_{n}\right)$, for which we have only the weak bound $n K_{l}$, occurs associated with the power $w^{l}$ in the exponent of $Q_{r+1}^{(2)}\left(w ; S_{n}\right)$. Writing

$$
\frac{d^{s}}{d z^{s}} Q_{r+1}^{(2)}\left(z ; S_{n}\right)=P_{s}(n, z) Q_{r+1}^{(2)}\left(z ; S_{n}\right)
$$

the monomials that make up $P_{s}(n, z)$ thus have coefficients of magnitude $n^{l}$ associated with powers $z^{m}$ with $m \geq(2 l-(s-l))_{+}=(3 l-s)_{+}$, so that they are themselves of magnitude at most $O\left(n^{l-(3 l-s)_{+} / 3}\right)=O\left(n^{s / 3}\right)$ in $\left|\theta^{\prime}\right| \leq n^{-1 / 3}$. Taking $s=L_{r}+1$ and $r \geq 2, m=0$ requires that $l \leq r-1$, and $l \geq r$ entails $m \geq 2$, so that, for $r \geq 2$ and $|\theta| \leq \theta_{0}$,

$$
\sup _{\left|\theta^{\prime}\right| \leq|\theta|}\left|\frac{d^{L_{r}+1}}{d z^{L_{r}+1}} Q_{r+1}^{(2)}\left(z ; S_{n}\right)\right|_{z=e^{i \theta^{\prime}-1}} \leq k_{3} n^{r-1}\left(1+n|\theta|^{2}\right),
$$

with $k_{3}=k_{3}\left(K^{(n)}, K_{1}, \ldots, K_{r+1}\right)$. If $|\theta| \geq n^{-1 / 2}$, it follows that the bound in (8.14) is at most $2 k_{3}\left\{\left(L_{r}+1\right) !\right\}^{-1} n^{r}|\theta|^{3 r}$; if $|\theta| \leq n^{-1 / 2}$, the bound is at most $2 k_{3}\left\{\left(L_{r}+1\right) !\right\}^{-1} n|\theta|^{r+2}$. Combining this with (8.13), we have established that for $|\theta| \leq n^{-1 / 3}$ and $r \geq 2$, we have

$$
\left|\phi_{\mu}(\theta) \exp \left\{-\lambda\left(e^{i \theta}-1\right)\right\}-\widetilde{A}^{(r)}(\theta)\right| \leq k_{4} n|\theta|^{r+1+\delta}\left(1+\left(n|\theta|^{2}\right)^{r-1}\right)
$$

where $k_{4}=k_{4}\left(K^{(n)}, K_{1}, \ldots, K_{r+1}\right)$. This gives

$$
\begin{gathered}
\gamma_{1}=n k_{4}, \quad t_{1}=r+1+\delta, \quad \gamma_{2}=n^{r} k_{4}, \quad t_{2}=3 r-1+\delta \\
M=2, \quad \rho=2 \lambda / \pi^{2}, \quad \varepsilon=0 \quad \text { and } \quad \theta_{0}=n^{-1 / 3}
\end{gathered}
$$

in Proposition 3.1, together with $\eta=C e^{-n^{1 / 3} \rho^{\prime}}$ from the bounds (8.7) and (8.8). Applying Corollary 3.2, and using the tail properties of the Poisson-Charlier measures (5.5), the theorem follows for $r \geq 2$.

For $r=1$, the bound in (8.14) is easily of order $|\theta|^{2}$, giving a bound in (8.15) of $k_{4}^{\prime}\left(n|\theta|^{2+\delta}+|\theta|^{2}\right)$. This leads to the choices

$$
\begin{gathered}
\gamma_{1}=n k_{4}^{\prime}, \quad t_{1}=2+\delta, \quad \gamma_{2}=k_{4}^{\prime}, \quad t_{2}=2 d \\
M=2, \quad \rho=2 \lambda / \pi^{2}, \quad \varepsilon=0 \quad \text { and } \quad \theta_{0}=n^{-1 /(2+\delta)}
\end{gathered}
$$

in Proposition 3.1, together with $\eta=C e^{-n^{\delta /(2+\delta)} \rho^{\prime}}$, and the remainder of the proof is as before.

Remark. A total variation bound for the error, of the slightly larger order $O\left(n^{-(r-1+\delta) / 2} \sqrt{\log n}\right)$, can also be deduced from Corollary 3.2, by taking $a=\lfloor\lambda-k \sqrt{\lambda \log \lambda}\rfloor$ and $b=\lceil\lambda+$ $k \sqrt{\lambda \log \lambda}\rceil$, for suitable choice of $k=k_{r}$.

8.2. Analytic combinatorial schemes. An extremely interesting range of applications is to be found in the paper of Hwang (1999). His conditions are motivated by examples from combinatorics, in which generating functions are natural tools. He works in an asymptotic setting, assuming that $X_{n}$ is a random variable whose probability generating function $R_{n}$ is of the form

$$
R_{n}(z)=z^{h}\left(g(z)+\varepsilon_{n}(z)\right) e^{\lambda(z-1)},
$$


where $h$ is a non-negative integer, and both $g$ and $\varepsilon_{n}$ are analytic in a closed disc of radius $\eta>1$. As $n \rightarrow \infty$, he assumes that $\lambda \rightarrow \infty$ and that $\sup _{z:|z| \leq \eta}\left|\varepsilon_{n}(z)\right| \leq K \lambda^{-1}$, uniformly in $n$. He then proves a number of results describing the accuracy of the approximation of $P_{X_{n}-h}$ by $\operatorname{Po}\left(\lambda+g^{\prime}(1)\right)$.

Under his conditions, it is immediate that we can write

$$
g(z)=\sum_{j \geq 0} g_{j}(z-1)^{j} \quad \text { and } \quad \varepsilon_{n}(z)=\sum_{j \geq 0} \varepsilon_{n j}(z-1)^{j}
$$

for $|z|<\eta-1$, with

$$
\left|g_{j}\right| \leq k_{g}(\eta-1)^{-j} \text { and }\left|\varepsilon_{n j}\right| \leq \lambda^{-1} k_{\varepsilon}(\eta-1)^{-j}
$$

for all $j \geq 0$. Hence $X:=X_{n}-h$ has characteristic function of the form $\psi^{(n)} p_{\lambda}$, where

$$
\psi^{(n)}(\theta)=g\left(e^{i \theta}\right)+\varepsilon_{n}\left(e^{i \theta}\right),
$$

and hence, for any $r \in \mathbb{N}_{0}$,

$$
\left|\psi^{(n)}(\theta)-\tilde{\psi}_{r}^{(n)}(\theta)\right| \leq K_{r 1}|\theta|^{r+1}, \quad|\theta| \leq(\eta-1) / 2,
$$

with $\tilde{\psi}_{r}^{(n)}$ defined as in (2.4), taking $\tilde{a}_{j}^{(n)}=g_{j}+\varepsilon_{n j}$; note that the constant $K_{r 1}$ can indeed be taken to be uniform for all $n$. Since also $g$ and $\varepsilon_{n}$ are both uniformly bounded on the unit circle, and since $\tilde{\psi}_{r}^{(n)}$ is bounded (uniformly in $n$ ) for $|\theta| \leq \pi$, it is clear that (8.18) can be extended to all $|\theta| \leq \pi$, albeit with a different uniform constant $K_{r 1}^{\prime}$, so that (2.5) holds with $\delta=1$ for any $r \in \mathbb{N}_{0}$. Thus Corollary 4.1 (with $R_{\lambda}=\operatorname{Po}(\lambda)$ and $h(\lambda)=2 \lambda / \pi^{2}$ ) and Theorem 5.1 can be applied with any choice of $r$, giving progressively more accurate approximations to $P_{X_{n}-h}$, as far as the $\lambda$-order is concerned, in terms of progressively more complicated perturbations of the Poisson distribution. These theorems are thus applicable to all the examples that Hwang considers, including the numbers of components (counted in various ways) in a wide class of logarithmic assemblies, multisets and selections.

For instance, Corollary 6.2 gives an approximation to $P_{X_{n}-h}$ by the mixture $Q_{m p}\left(\operatorname{Po}\left(\lambda^{\prime}\right)\right)$ with

$$
m:=\left\lfloor m_{n}-v_{n}\right\rfloor ; \quad p^{2}:=\left\langle m_{n}-v_{n}\right\rangle ; \quad \lambda^{\prime}:=\lambda+v_{n}-p(1-p),
$$

where $m_{n}:=g_{n}^{\prime}(1), v_{n}:=g_{n}^{\prime \prime}(1)+g_{n}^{\prime}(1)-\left\{g_{n}^{\prime}(1)\right\}^{2}$ and $g_{n}:=g+\varepsilon_{n}$. Hwang's approximation by $\operatorname{Po}\left(\lambda+g^{\prime}(1)\right)$ has asymptotically the same mean as ours (and as that of $X_{n}-h$ ), but a variance asymptotically differing by $\kappa:=g^{\prime \prime}(1)-\left\{g^{\prime}(1)\right\}^{2}$. As a consequence, Hwang's approximation has an error of larger asymptotic order, in which the quantity $\kappa$ appears; for instance, for Kolmogorov distance, his Theorem 1 gives an error of order $O\left(\lambda^{-1}\right)$, whereas that from Corollary 6.2 is of order $O\left(\lambda^{-3 / 2}\right)$.

Although our Poisson expansion theorems are automatically applicable under Hwang's conditions, they also apply to examples that do not satisfy his conditions: the simple example at the end of Section 2 is one such. Conversely, Hwang's Theorem 2, which establishes Poisson approximation in the lower tail with good relative accuracy, cannot be proved using only our conditions; the conclusion would not be true, for instance, in the example just mentioned.

Note also that Hwang examines problems from combinatorial settings in which approximation is not by Poisson distributions: he has examples concerning the Bessel family of distributions,

$$
B(\lambda)\{j\}:=L(\lambda)^{-1} \frac{\lambda^{j}}{j !(j-1) !}, \quad j \in \mathbb{N},
$$


for the appropriate choice of normalizing constant $L(\lambda)$. This family also satisfies the conditions for $R_{\lambda}$ of Section 3, and we could apply Corollary 4.2 to obtain slightly sharper approximations than his within the translated Bessel family, or Corollary 4.1 to obtain asymptotically more accurate expansions.

8.3. Prime divisors. The numbers of prime divisors of a positive integer $n$, counted either with $(\Omega(n))$ or without $(\omega(n))$ multiplicity, can also be treated by these methods, since excellent information is available about their generating functions. For our purposes, we use only the shortest expansion, taken from Tenenbaum (1995, Theorems II.6.1 and 6.2). One finds that, for $N_{n}$ uniformly distributed on $\{1,2, \ldots, n\}$, the characteristic functions of the we have

$$
\begin{aligned}
& \mathbb{E}\left\{e^{i \theta \omega\left(N_{n}\right)}\right\}=p_{\log \log n}(\theta)\left\{\Phi_{1}\left(e^{i \theta}-1\right)+\eta_{1}(\theta)\right\} \\
& \mathbb{E}\left\{e^{i \theta \Omega\left(N_{n}\right)}\right\}=p_{\log \log n}(\theta)\left\{\Phi_{2}\left(e^{i \theta}-1\right)+\eta_{2}(\theta)\right\}
\end{aligned}
$$

where $\left|\eta_{s}(\theta)\right| \leq C_{s} / \log n, s=1,2$, for some constants $C_{1}$ and $C_{2}$, and

$$
\begin{aligned}
\Phi_{1}(w) & :=\frac{1}{\Gamma(1+w)} \prod_{q}\left(1+\frac{w}{q}\right)\left(1-\frac{1}{q}\right)^{w} \\
\Phi_{2}(w) & :=\frac{1}{\Gamma(1+w)} \prod_{q}\left(1-\frac{w}{q-1}\right)^{-1}\left(1-\frac{1}{q}\right)^{w}
\end{aligned}
$$

$q$ running here over prime numbers. These expansions were established and used by Rényi and Turán (1958) in their proof of the Erdős-Kac Theorem, but they are also sketched by Selberg (1954). We refer to Kowalski and Nikeghbali (2009) for the structural interpretation of the two factors in these functions, with $1 / \Gamma(1+w)$ being related to the number of cycles of large random permutations.

Let $\tilde{a}_{l s}, s=1,2$, denote the Taylor coefficients of the functions $\Phi_{s}(w)$ as power series in $w$ (around $w=0$, which corresponds to $\theta=0$ ). By analyticity near 0 , it follows that, for any $r$, we have

$$
\left|\Phi_{s}(w)-1-\sum_{l=1}^{r} \tilde{a}_{l s} w^{l}\right| \leq C_{r s}|w|^{r+1} ; \quad\left|\Phi_{s}^{\prime \prime}(w)-\sum_{l=2}^{r} \tilde{a}_{l s} l(l-1) w^{l-2}\right| \leq C_{r s}^{\prime}|w|^{r-1},
$$

for suitable constants $C_{r s}, C_{r s}^{\prime}$ and for $|w| \leq 2$. In order to approximate the distributions $P_{\omega\left(N_{n}\right)}$ and $P_{\Omega\left(N_{n}\right)}$, we define the measures $\nu_{r}^{(s)}$ by

$$
\nu_{r}^{(s)}\{j\}:=\operatorname{Po}(\log \log n)\{j\}\left(1+\sum_{l=1}^{r}(-1)^{l} \tilde{a}_{l s} C_{l}(j ; \log \log n)\right),
$$

and invoke Propositions 3.1 and 3.4 together with (3.5); this leads to the following conclusion, which refines the Erdős-Kac theorem. 
Theorem 8.2. For the measures $\nu_{r}^{(s)}$ defined above, we have

$$
\begin{aligned}
& d_{\mathrm{loc}}\left(P_{\omega\left(N_{n}\right)}, \nu_{r}^{(1)}\right) \leq \alpha_{1, r+1}^{\prime} C_{r 1}(\log \log n)^{-1-r / 2}+\tilde{\alpha}_{1} C_{1} / \log n ; \\
&\left\|P_{\omega\left(N_{n}\right)}-\nu_{r}^{(1)}\right\| \leq 2 \alpha^{\prime}\left(r+1, \pi^{2} / 2\right) C_{r 1}^{\prime}\left(1+\frac{2}{r}\right)(\log \log n)^{-(r+1) / 2} \\
&+\widetilde{C}_{1} \log \log n / \log n ; \\
& d_{\mathrm{loc}}\left(P_{\Omega\left(N_{n}\right)}, \nu_{r}^{(2)}\right) \leq \alpha_{1, r+1}^{\prime} C_{r 2}(\log \log n)^{-1-r / 2}+\tilde{\alpha}_{1} C_{2} / \log n ; \\
&\left\|P_{\Omega\left(N_{n}\right)}-\nu_{r}^{(2)}\right\| \leq 2 \alpha^{\prime}\left(r+1, \pi^{2} / 2\right) C_{r 2}^{\prime}\left(1+\frac{2}{r}\right)(\log \log n)^{-(r+1) / 2} \\
&+\widetilde{C}_{2} \log \log n / \log n,
\end{aligned}
$$

for suitable constants $\widetilde{C}_{1}$ and $\widetilde{C}_{2}$, and with $\alpha_{1 l}^{\prime}$ as defined in (5.12).

Remark. As far as we know, total variation approximation was first considered in this context by Harper (2009), who proved a bound with error of size $1 /(\log \log n$ ) (for a truncated version of $\omega(n)$, counting only prime divisors of size up to $\left.n^{1 /\left(3(\log \log n)^{2}\right)}\right)$, and deduced explicit bounds in Kolmogorov distance.

To indicate what this means in concrete terms for number theory readers, consider the case of $\omega(n)$ for $r=1$. Taylor expansion gives

$$
\Phi_{1}(w)=1+B_{1} w+O\left(w^{2}\right)
$$

as $w \rightarrow 0$, where $B_{1} \approx 0.26149721$ is the Mertens constant, i.e., the real number such that

$$
\sum_{\substack{q \leq x \\ q \text { prime }}} \frac{1}{q}=\log \log x+B_{1}+o(1)
$$

as $x \rightarrow+\infty$. An application of Theorem 8.2 gives

$$
\begin{aligned}
\left|\frac{1}{n}\right|\{k \leq n \mid \omega(n) \in A\}\left|-\nu_{1}^{(1)}\{A\}\right| & \leq \frac{1}{2}\left\|P_{\omega\left(N_{n}\right)}-\nu_{1}^{(1)}\right\| \\
& =O\left(\frac{1}{\log \log n}\right),
\end{aligned}
$$

for any set $A$ of positive integers, where

$$
\nu_{1}^{(1)}\{j\}=\operatorname{Po}(\log \log n)\{j\}\left(1-B_{1}\left\{1-\frac{j}{\log \log n}\right\}\right) .
$$

Higher expansions could be computed in much the same way.

Alternatively, a more accurate approximation is available from Corollary 6.2, while staying within the realm of (translated) Poisson distributions. For this, we compute the expansion of $\Phi_{1}$ to order 2 , obtaining (after some calculations) that

$$
\Phi_{1}(w)=1+\tilde{a}_{1} w+\tilde{a}_{2} w^{2}+O\left(w^{3}\right), \quad \text { as } w \rightarrow 0,
$$

where

$$
\tilde{a}_{1}:=B_{1} ; \quad \tilde{a}_{2}:=\frac{B_{1}^{2}}{2}-\frac{\pi^{2}}{12}-\frac{1}{2} \sum_{q \text { prime }} \frac{1}{q^{2}}
$$

(use $1 / \Gamma(1+w)=1+\gamma w+\left(\gamma^{2} / 2-\pi^{2} / 12\right) w^{2}+O\left(w^{3}\right)$, as well as the Mertens identity

$$
\gamma+\sum_{q \text { prime }}\left(\frac{1}{q}+\log \left(1-\frac{1}{q}\right)\right)=B_{1},
$$

and expand every term in the Euler product). This corresponds to (2.5), since $w=e^{i \theta}-1$. 
We can then apply Corollary 6.2 to get the translated Poisson approximation $Q_{m p}\left(\operatorname{Po}\left(\lambda^{\prime}\right)\right)$, with parameters calculated using (6.1). With

$$
x:=\tilde{a}_{1}^{2}-2 \tilde{a}_{2}=\frac{\pi^{2}}{6}+\sum_{q \text { prime }} \frac{1}{q^{2}} \approx 2.0971815,
$$

this gives

$$
\begin{aligned}
p & =\sqrt{\langle x\rangle} \approx 0.31173945 ; \quad m=2 ; \\
\lambda^{\prime} & =\log \log n+B_{1}-x-p(1-p) \approx \log \log n-2.0502422 .
\end{aligned}
$$

Thus, for any positive integer $n$ and any set $A$ of positive integers, we have

$$
\begin{aligned}
\left|\frac{1}{n}\right|\{k \leq n \mid \omega(n) \in A\} \mid-\left\{p \operatorname{Po}\left(\lambda^{\prime}\right)\{A-3\}+(1-p) \operatorname{Po}\left(\lambda^{\prime}\right)\{A\right. & -2\}\} \mid \\
& =O\left(\frac{1}{(\log \log n)^{3 / 2}}\right) .
\end{aligned}
$$

Similar results hold for $\Omega(n)$, where one obtains the following approximate values for the quantities $p, m, \lambda^{\prime}$ :

$$
p \approx 0.5195 ; \quad m=0 ; \quad \lambda^{\prime} \approx \log \log n+0.5152 .
$$

\section{REFERENCES}

[1] A. D. Barbour \& V. ČekanaviČıus (2002) Total variation asymptotics for sums of independent integer random variables. Ann. Probab. 30, 509-545.

[2] T. S. Chinara (1978) An introduction to orthogonal polynomials. Gordon and Breach, New York.

[3] F. Chung \& L. Lu (2006) Concentration inequalities and martingale inequalities: a survey. Internet Math. 3, 79-127.

[4] A. J. Harper (2009) Two new proofs of the Erdős-Kac Theorem, with bound on the rate of convergence, by Stein's method for distributional approximations. Math. Proc. Cam. Phil. Soc. $147,95-114$.

[5] H.-K. Hwang (1999) Asymptotics of Poisson approximation to random discrete distributions: an analytic approach. Adv. Appl. Prob. 31, 448-491.

[6] J. Jacod, E. Kowalski \& A. Nikeghbali (2008) Mod-Gaussian convergence: new limit theorems in probability and number theory, to appear in Forum Math.; see also arXiv:0807.4739.

[7] E. Kowalski \& A. Nikeghbali (2009) Mod-Poisson convergence in probability and number theory. arXiv:0905.0318.

[8] V. V. Petrov (1975) Limit theorems of probability theory. Oxford University Press, Oxford.

[9] E. L. Presman (1983) Approximation of binomial distributions by infinitely divisible ones. Theor. Prob. Appl. 28, 393-403.

[10] A. RÉnYi \& P. Turán (1958) On a theorem of Erdős-Kac. Acta Arith. 4, 71-84.

[11] A. Selberg (1954) Note on the paper by L.G. Sathe. J. Indian Math. Soc. 18 83-87.

[12] G. Tenenbaum (1995) Introduction à la théorie analytique et probabiliste des nombres. Société Mathématique de France.

InStitut FÜr Mathematik, Universität ZÜrICh, WinterthuRERTRASSE 190, CH-8057 ZÜRICH, SWITZERLAND

E-mail address: a.d.barbour@math.uzh.ch

ETH Zurich, D-MATH, RÄmistrasse 101, 8092 ZÜRICH, Switzerland

E-mail address: kowalski@math.ethz.ch

Institut FÜr Mathematik, Universität ZÜRICH, WinterthURERTRAsse 190, CH-8057 ZÜRICH, SWITZERLAND

E-mail address: ashkan.nikeghbali@math.uzh.ch 